IR-11-020

\title{
Oligomorphic dynamics for analyzing the quantitative genetics of adaptive speciation
}

Akira Sasaki (sasaki_akira@soken.ac.jp)

Ulf Dieckmann (dieckmann@iiasa.ac.at)

\section{Approved by \\ Detlof Von Winterfeldt \\ Director}

July 2011 Institute, its National Member Organizations, or other organizations supporting the work. 


\section{Contents}

Abstract. 
Effects of mutation on evolutionary branching ..................................................... 19

Analytical results........................................................................................... 20

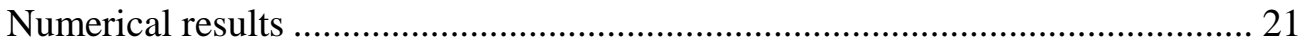

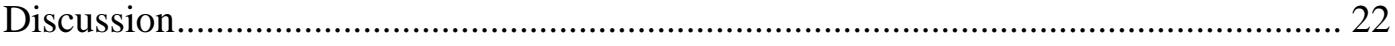

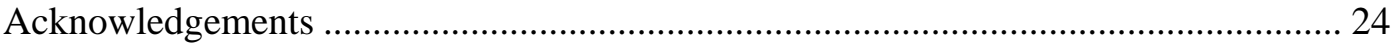

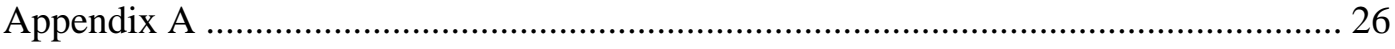

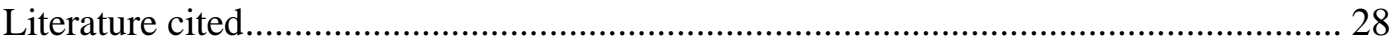

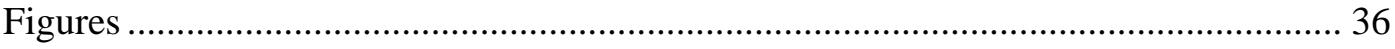




\title{
Oligomorphic dynamics for analyzing the quantitative genetics of adaptive speciation
}

\author{
Akira Sasaki ${ }^{1,2,3}$ \\ ${ }^{1}$ Department of Evolutionary Study of Biosystems, The Graduate University for Advanced \\ Studies (Sokendai), Hayama, Kanagawa 240-0193, Japan \\ Email: sasaki_akira@soken.ac.jp, Tel: +81-46-858-1537, Fax: +81-46-858-1544 \\ 2 Evolution and Ecology Program, International Institute for Applied Systems Analysis, \\ A-2361 Laxenburg, Austria \\ ${ }^{3}$ PRESTO, Japan Science and Technology Agency, 4-1-8 Honcho Kawaguchi, Saitama, Ja- \\ pan \\ and \\ Ulf Dieckmann ${ }^{2}$ \\ 2 Evolution and Ecology Program, International Institute for Applied Systems Analysis, \\ A-2361 Laxenburg, Austria \\ Email: dieckmann@iiasa.ac.at
}

\begin{abstract}
Ecological interaction, including competition for resources, often causes frequency-dependent disruptive selection, which, when accompanied by reproductive isolation, may act as driving forces of adaptive speciation. While adaptive dynamics models have added new perspectives to our understanding of the ecological dimensions of speciation processes, it remains an open question how best to incorporate and analyze genetic detail in such models. Conventional approaches, based on quantitative genetics theory, typically assume a unimodal character distribution and examine how its moments change over time. Such approximations inevitably fail when a character distribution becomes multimodal. Here, we propose a new approximation, oligomorphic dynamics, to the quantitative genetics of populations that include several morphs and that therefore exhibit multiple peaks in their character distribution. To this end, we first decompose the character distribution into a sum of unimodal distributions corresponding to individual morphs. Characterizing these morphs by their frequency (fraction of individuals belonging to each morph), position (mean character of each morph), and width (standard deviation of each morph), we then derive the coupled eco-evolutionary dynamics of morphs through a double Taylor expansion. We show that the demographic, convergence, and evolutionary stability of a population's character distribution correspond, respectively, to the asymptotic stability of frequencies, positions, and widths under the oligomorphic dynamics introduced here. As first applications of oligomorphic dynamics theory, we analytically derive the effects (a) of the strength of disruptive selection on waiting times until speciation, (b) of
\end{abstract}


mutation on conditions for speciation, and (c) of the fourth moments of competition kernels on patterns of speciation.

Keywords: adaptive dynamics, quantitative genetics theory, moment dynamics, adaptive speciation, evolutionarily stable strategy, convergence stability

Classification code: MSC 92 (Biology and other natural sciences) -- 92D10 (Genetics), 92D15 (Problems related to evolution), or 92D40 (Ecology).

\section{Introduction}

Quantitative genetics theory has been successful in analyzing a wide variety of evolutionary processes, including trait shifts under directional, disruptive, or temporally fluctuating natural or artificial selection (Lande 1979; Bulmer 1992; Falconer 1996); mechanisms for maintaining standing genetic variation by mutation-selection balance, fluctuating selection, or heterosis (Kimura and Crow 1964; Bulmer 1972; Lande 1975; Felsenstein 1976; Ellner and Hairston 1994; Ellner and Sasaki 1996; Kondrashov and Yampolsky 1996; Sasaki and Ellner 1997); as well as escalations of male ornaments and female preferences through runaway selection (Lande 1981; Lande and Kirkpatrick 1988; Iwasa et al. 1991).

A limitation in many applications of quantitative genetics theory arises from a focus on unimodal character distributions, which simplifies the derivation of equations for the temporal change of population genetics quantities. To justify the required moment closures, character distributions have been assumed to be of Gaussian shape (e.g., Lande 1979) or to be narrowly localized around a single mean (e.g., Iwasa et al. 1991). Moreover, many applications of quantitative genetics theory assume genetic variances and covariances to be constant, to make the analyzed models more tractable. Such approximations must therefore fail once the distribution of a quantitative character starts becoming bimodal. The latter is expected under frequency-dependent disruptive selection. Such selection can arise from a great variety of ecological processes, including symmetric intraspecific competition (Metz et al. 1996; Sasaki 1997; Doebeli 1996a, 1996b; Dieckmann and Doebeli 1999), asymmetric intraspecific competition (Kisdi 1999; Doebeli and Dieckmann 2000; Kisdi et al. 2001), interspecific competition (Law et al. 1997; Kisdi and Geritz 2001), resource specialization (Meszéna et al. 1997; Geritz et al. 1998; Day 2000; Kisdi 2001; Schreiber and Tobiason 2003; Egas et al. 2004, 2005), temporally fluctuating selection with storage effect (Ellner and Hairston 1994; Sasaki and Ellner 1995, 1997; Ellner and Sasaki 1996), ontogenetic niche shifts (Claessen and Dieckmann 2002), mixotrophy (Troost et al. 2005), phenotypic plasticity (Sasaki and Ellner 1995; Sasaki and de Jong 1999; Van Dooren and Leimar 2003; Ernande and Dieckmann 2004; Leimar 2005), dispersal evolution (Levin et al. 1984; Cohen and Levin 1991; Ludwig and Levin 1991; Doebeli and Ruxton 1997; Johst et al. 1999; Parvinen 1999; Mathias et al. 
2001; Parvinen and Egas 2004), mutation evolution (Haraguchi and Sasaki 1996), mutualism (Doebeli and Dieckmann 2000; Law et al. 2001; Ferdy et al. 2002; Ferrière et al. 2002; Day and Young 2004), emergent cooperation (Doebeli et al. 2004), predator-prey interactions (Brown and Pavlovic 1992; Van der Laan and Hogeweg 1995; Doebeli and Dieckmann 2000; Bowers et al. 2003), cannibalism (Dercole 2003), evolution of virulence (Boots et al. 2004; Kamo et al. 2007), host-parasite interactions (Haraguchi and Sasaki 1996, 1997; Boots and Haraguchi 1999; Sasaki and Godfray 1999; Koella and Doebeli 1999; Regoes et al. 2000; Gudelj et al. 2004), sex-ratio evolution (Metz et al. 1992; Reuter et al. 2004), evolution of selfing (Cheptou and Mathias 2001; De Jong and Geritz 2001), evolution of mating traits (Van Doorn et al. 2001, 2004), evolution of anisogamy (Matsuda and Abrams 1999; Maire et al. 2001), evolution of cytoplasmic inheritance (Iwanaga and Sasaki 2004), seed-size evolution (Rees and Westoby 1997; Geritz et al. 1999; Mathias and Kisdi 2002), microbial cross-feeding (Doebeli 2002), prebiotic evolution (Meszéna and Szathmáry 2001), resource competition among digital organisms (Chow et al. 2004), and evolutionary community assembly (Jansen and Mulder 1999; Bonsall et al. 2004; Loeuille and Loreau 2005). These processes are important for understanding adaptive speciation and many other processes involving frequency-dependent interactions within or between species. Analyses of character distributions with an evolutionarily variable number of modes have therefore relied on numerical investigations or on game theory and adaptive dynamics theory (e.g., Eshel and Motro 1981; Eshel 1983; Ludwig and Levin 1991; Sasaki and Ellner 1995, 1997; Dieckmann and Law 1996; Sasaki 1997; Dieckmann and Doebeli 1999; Sasaki and Godfray 1999; Doebeli and Dieckmann 2000, 2003). The latter have to assume a minimal degree of population genetic complexity and often do not account for polymorphic genetic variation around a distribution's modes.

In this study, we propose a new approximation, oligomorphic dynamics, to describe the quantitative genetic dynamics of asexually reproducing populations that contain multiple morphs and therefore exhibit multiple peaks in their character distribution. The main idea of this approximation is simple and our approach proceeds in three steps: we (1) decompose a population's character distribution into a sum of single-peaked distributions, each corresponding to one morph; (2) characterize each morph by its frequency (fraction of individuals belonging to the morph), position (mean character of the morph), and width (standard deviation of the morph); and (3) derive the equations that govern the dynamics of these quantities.

A central purpose of the oligomorphic approximation is to analyze the transitions through which an evolving character distribution becomes divided into several morphs and reaches a multimodal stationary state. We derive the approximate moment dynamics in terms of the frequencies, mean phenotypes, and variances of the morphs. Assuming that the widths of morphs are small relative to their distances, we derive the equations for the first three mo- 
ments through a novel approach of double Taylor expansion. Importantly, the distances between morphs do not have to be small for the oligomorphic approximation to be accurate.

Our theory builds on the dynamics of mean quantitative characters pioneered by Lande (1976, 1979, 1981, 1982), who showed that changes in a unimodal distribution's mean character are proportional to the gradient of mean fitness as a function of mean character. Further work derived the dynamics of higher central moments (such as variance and skewness) and made analyses more mathematically tractable by focusing on small deviations of characters from a population's mean character (Barton and Turelli 1991). Another important extension of Lande's work occurred through the inclusion of frequency-dependent selection (Iwasa et al. 1991; Abrams et al. 1993; Vincent et al. 1993). If restricted to unimodal character distributions, our oligomorphic approximation reduces to the theory of Taylor and Day (1997). Our oligomorphic approximation is also related to character-displacement models (Roughgarden 1972, 1976; Bulmer 1974; Slatkin 1980; Matessi and Jayakar 1981; Taper and Case 1985). These earlier models, however, assumed either a fixed variance for each species (e.g., Roughgarden 1976), a fixed Gaussian shape of each species’ character distribution (e.g., Slatkin 1980), or simple major-locus inheritance (based, e.g., on single-locus two-allele genetics; Bulmer 1974). Reasons why character distributions are expected to exhibit distinctly separated peaks were elucidated by May (1973), Sasaki and Ellner (1995), Sasaki (1997), Gyllenberg and Meszéna (2005), Doebeli et al. (2007), Pigolotti et al. (2007, 2009), Leimar et al. (2008), and Fort et al. (2009).

To illustrate the utility of the oligomorphic approximation, we consider evolutionary processes driven by resource competition (MacArthur 1970; Rosenzweig 1978; Roughgarden 1972). In these models, individuals with similar phenotypes compete more intensely than phenotypically distant individuals. Two antagonistic selection pressures then need to be considered: the first results from frequency-dependent disruptive selection due resource competition, and the second from frequency-independent stabilizing selection towards an optimal phenotype at which, in the absence of competition, the resource is most abundant. Under these conditions, disruptive selection may cause the character distribution to split into several morphs. To derive the moment dynamics for each morph, it is necessary to evaluate the competitive effects between individuals belonging to different morphs. This is why a standard moment-closure approach based on Taylor expansions assuming small character deviations around a common mean fails for processes allowing multimodal character distributions. By contrast, the oligomorphic dynamics proposed here successfully overcome this limitation by expanding the phenotypic effect of a competitor around the mean of the morph to which the competitor belongs, rather than around the mean of the morph to which the focal individual belongs. 


\section{Model description}

We call a character distribution oligomorphic if it comprises a finite number of distinct peaks. Below, we first derive the dynamics of an oligomorphic character distribution and then approximate these in terms of moment equations. Throughout, we illustrate our approach by using a continuous-time model of character-mediated competition.

\subsection{Resource competition}

We consider a continuum of ecological characters $x$ describing the peak of an organism's resource utilization spectrum along a one-dimensional niche space. The resource abundance at niche position $x$ is denoted by $K(x)$, and the density of individuals with character $x$ is denoted by $N(x)$. The competition coefficients between individuals with characters $x$ and $y$ are given by $a(x-y)$. Because of the formal role it plays in the integral in eq. (1), the function $a(d)$ is called the competition kernel. It is assumed to attain its maximum at $d=0$, implying that competition is strongest between individuals with identical characters, and to decrease monotonically towards 0 as $|d|$ increases. We also assume that competition is symmetric, so that $a(x-y)=a(y-x)$ for all $x$ and $y$.

The dynamics of $N(x)$ are thus given by Lotka-Volterra competition equations for a continuum of characters,

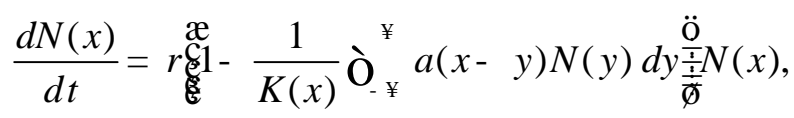

where $r$ denotes the intrinsic growth rate. The carrying capacity $K(x)$ is usually assumed to be unimodal around an optimum $x=0$, where the resource is most abundant. Without loss of generality, we assume that $a(0)=1$ and $K(0)=1$. By definition, $a \notin(0)=K(0)=0$. For the sake of brevity, below we leave out the integration limits shown in eq. (1).

We denote the total population density by $\bar{N}=$ ò $N(x) d x$. The dynamics of $\bar{N}$ are obtained by integrating both sides of eq. (1),

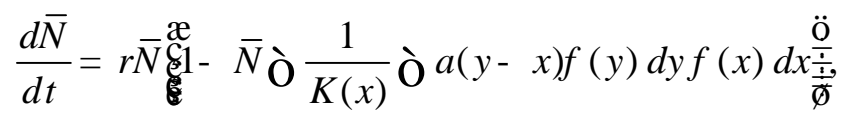

where $f(x)=N(x) / \bar{N}$ is the relative frequency of character $x$.

\subsection{Character dynamics}

The dynamics of the character distribution $f(x)=N(x) / \bar{N}$ are obtained by applying the chain rule and using eqs. (1) and (2), 


$$
\begin{aligned}
& \frac{d f}{d t}=\frac{1}{r \bar{N}} \frac{d}{d t} \frac{N(x)}{\bar{N}}=\frac{1}{r \bar{N}} \sum_{\mathbb{Q}}^{\circledR} \frac{1}{N(x)} \frac{d N(x)}{d t}-\frac{1}{\bar{N}} \frac{d \bar{N}}{d t} \frac{\ddot{0}}{\dot{\vec{\Xi}}} f(x)
\end{aligned}
$$

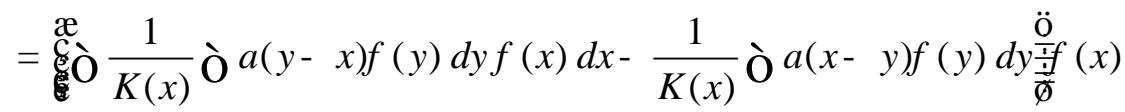

$$
\begin{aligned}
& =(w(x)-\bar{w}) f(x),
\end{aligned}
$$

where time is rescaled as $t=r \mathrm{O}_{0}^{t} \bar{N}(t \Phi d t \Phi$ to eliminate the dependence on total population density in the frequency dynamics. Thus, the frequency $f(x)$ of a character $x$ changes according to its fitness

$$
w(x)=1-\frac{1}{K(x)} \mathbf{0} a(x-y) f(y) d y,
$$

which depends on the population's character distribution $f$, making selection frequency-dependent. The population's mean fitness is denoted by $\bar{w}=$ ò $w(x) f(x) d x$. For a uniform competition kernel, $a(d)=1$ for all $d$, the fitness $w(x)$ is frequency-independent and stabilizing around $x=0$, where the carrying capacity $K(x)$ is largest. For sufficiently narrow competition kernels, the fitness landscape has a valley where the character distribution $f$ is peaked, implying frequency-dependent disruptive selection. The dynamics of $f$ are governed by the interplay between these selective forces.

\section{Results}

In the following, we introduce oligomorphic dynamics to describe how a population's character distribution may split into several modes under the influence of frequency-dependent disruptive selection, and how these modes and their shapes are expected to change over time. We then examine the conditions for such splits, which can be seen as describing sympatric speciation and/or character displacement in asexual populations. Next, we discuss the relationship of these conditions with three key stability concepts: demographic stability, convergence stability, and evolutionary stability. We then investigate the effects of mutation, and of the shape of competition kernels and resource distributions, on these conditions, on the possible patterns of speciation, and on the expected times to speciation.

\subsection{Oligomorphic dynamics}

We assume that the character distribution $f(x)$ consists of a few morphs $i=1,2, \mathrm{~L}, n$ ("a few" in English = "oligo" in Latin). These morphs have relative frequencies $p_{i}$, with å ${ }_{i=1}^{n} p_{i}=1$, so that

$$
f(x)=\stackrel{\circ}{\mathrm{a}}{ }_{i=1}^{n} p_{i} f_{i}(x) .
$$

For the sake of brevity, below we leave out the summation limits shown in eq. (5). Each morph $i$ can be regarded as a quasispecies, characterized by its character distribution $f_{i}(x)$, 
with $\mathrm{o} f_{i}(x) d x=1$. We define the dynamics of the frequencies $p_{i}$ so that for each $x$ the contribution of morphs to $d f(x) / d t$ is proportional to their contribution to $f(x)$,

$$
\frac{d p_{i} f_{i}(x)}{d t}=\frac{p_{i} f_{i}(x)}{f(x)} \frac{d f(x)}{d t} .
$$

Integrating this equation over all characters $x$ and using eq. (3), we obtain

$$
\frac{d p_{i}}{d t}=p_{i} \grave{\mathbf{O}} \frac{f_{i}(x)}{f(x)} \frac{d f(x)}{d t} d x=p_{i} \grave{\mathbf{O}}_{i}(x)(w(x)-\bar{w}) d x=\left(\bar{w}_{i}-\bar{w}\right) p_{i},
$$

where $\bar{w}_{i}=$ Ò $w(x) f_{i}(x) d x$ is the mean fitness of morph $i$.

To derive the dynamics of the character distribution $f_{i}(x)$ of morph $i$, we use the product rule, $\frac{d}{d t} p_{i} f_{i}(x)=p_{i} \frac{d}{d t} f_{i}(x)+f_{i}(x) \frac{d}{d t} p_{i}$, solve for $\frac{d}{d t} f_{i}(x)$, and then use eqs. (3), (6), and (7),

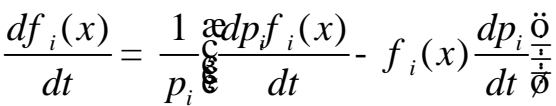

$$
\begin{aligned}
& =f_{i}(x)(w(x)-\bar{w})-f_{i}(x)\left(\bar{w}_{i}-\bar{w}\right) \\
& =\left(w(x)-\bar{w}_{i}\right) f_{i}(x) \text {. }
\end{aligned}
$$

\subsection{Moment approximation of oligomorphic dynamics}

We now derive the approximate dynamics of the first three moments of the character distributions of morphs, given by their frequencies $p_{i}$, means $\bar{x}_{i}=\mathrm{ò}_{x f_{i}}(x) d x$, and variances $V_{i}=$ ò $\left(x-\bar{x}_{i}\right)^{2} f_{i}(x) d x$. For this purpose, we first approximate the selection differentials $w(x)-\bar{w}_{i}$ in eq. (8).

\subsubsection{Approximation of selection differentials}

We denote by $x_{i}=x-\bar{x}_{i}$ the deviation of character $x$ from the mean character $\bar{x}_{i}$ of morph $i$. If $x$ is sufficiently close to $\bar{x}_{i}$, i.e., if $x_{i}$ is of order $e$, where $e$ is a sufficiently small positive constant, we can use a first Taylor expansion, of the interaction coefficients $a(x-y)$ around the morph means $y=\bar{x}_{j}$, to approximate the fitness $w(x)$ of character $x$ by

$$
\begin{aligned}
& w(x)=1-\frac{1}{K(x)} \stackrel{\circ}{~}_{j} p_{j} \grave{\mathbf{O}} a(x-y) f_{j}(y) d y
\end{aligned}
$$

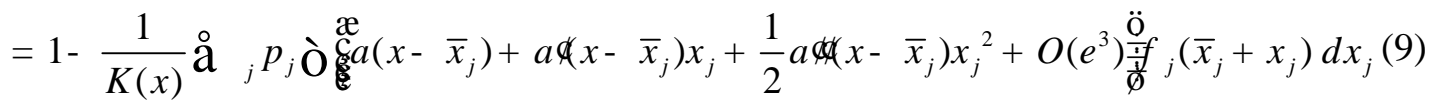

$$
\begin{aligned}
& =1-\frac{1}{K(x)} \stackrel{\circ}{\mathrm{a}}{ }_{j} p_{j} a\left(x-\bar{x}_{j}\right)-\frac{1}{2} \frac{1}{K(x)} \stackrel{\circ}{\mathrm{a}}{ }_{j} p_{j} a \phi\left(x-\bar{x}_{j}\right) V_{j}+O\left(e^{3}\right) \text {. }
\end{aligned}
$$

Furthermore, we can use a second Taylor expansion of the fitness $w(x)$ around the morph mean $x=\bar{x}_{i}$, 


$$
w(x)=w\left(\bar{x}_{i}\right)+\left.\frac{\prod w(x)}{\prod x}\right|_{x=\bar{x}_{i}} x_{i}+\left.\frac{1}{2} \frac{\prod^{2} w(x)}{\prod x^{2}}\right|_{x=\bar{x}_{i}} x_{i}^{2}+O\left(e^{3}\right) .
$$

Multiplying this equation with $f_{i}(x)$ and integrating over all $x$ yields

$$
\bar{w}_{i}=w\left(\bar{x}_{i}\right)+\left.\frac{1}{2} \frac{\rrbracket^{2} w(x)}{\llbracket x^{2}}\right|_{x=\bar{x}_{i}} V_{i}+O\left(e^{4}\right) .
$$

The selection differential for morph $i$ is obtained, up to second order in $e$, by subtracting eq. (11) from eq. (10),

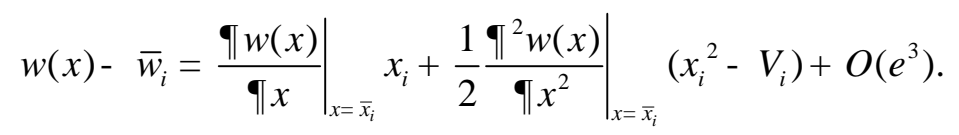

The two partial derivatives are obtained from eq. (9), in leading order of $e$, as

$$
\begin{aligned}
& \left.\frac{\llbracket w(x)}{\llbracket x}\right|_{x=\bar{x}_{i}}=-\stackrel{\circ}{\mathrm{a}}{ }_{j} p_{j}\left(a \Phi\left(\bar{x}_{i}-\bar{x}_{j}\right) v\left(\bar{x}_{i}\right)+a\left(\bar{x}_{i}-\bar{x}_{j}\right) v \Phi\left(\bar{x}_{i}\right)\right)+O\left(e^{2}\right),
\end{aligned}
$$

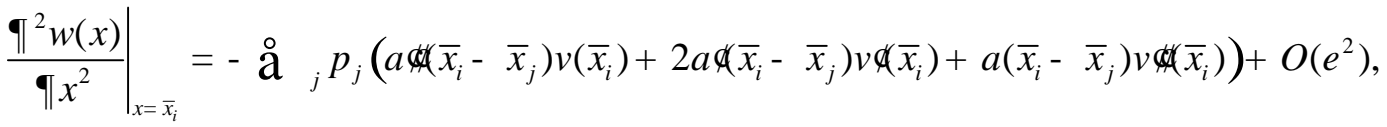

where $v$ is the inverse of carrying capacity, $v(x)=1 / K(x)$, which implies

$$
v \Phi(x)=-\frac{K \Phi(x)}{K(x)^{2}} \quad \text { and } \quad v \Phi(x)=\frac{2 K \Phi(x)^{2}}{K(x)^{3}}-\frac{K \Phi(x)}{K(x)^{2}} .
$$

The approximation provided by eqs. (12)-(15) is accurate if two conditions are met: (i) for all morphs $i$, the character distribution of that morph is sufficiently narrowly distributed around its mean, i.e., $\max _{i} \sqrt{V_{i}}$ is small (of order $e$ ); and (ii) for all morphs $i$ and $j$, the distance $d_{i j}=\bar{x}_{i}-\bar{x}_{j}$ is sufficiently larger than $\sqrt{V_{i}}$ and $\sqrt{V_{j}}$, ensuring multimodality. The first condition is required for the double Taylor expansion in eqs. (9) and (10), while the second condition is required, not for the derivation of those equations, but only for a natural decomposition of the character distribution in eq. (5). Combining these two requirements, the oligomorphic approximation is applicable whenever

$$
\frac{\max _{i} \sqrt{V_{i}}}{\min _{i j} d_{i j}}<e
$$

where $e^{\mathbb{\Phi}}$ is a sufficiently small positive constant.

\subsubsection{Dynamics of morph frequencies}

The growth rate of the frequency of morph $i$ is obtained from eq. (11), in leading order of $e$, as 


$$
\begin{aligned}
\bar{w}_{i}-\bar{w} & =w\left(\bar{x}_{i}\right)-\mathrm{a}_{k} w\left(\bar{x}_{k}\right) p_{k}+O\left(e^{2}\right) \\
& =\stackrel{\mathrm{a}}{k}_{k} p_{k} \frac{\mathrm{a}_{j} a\left(\bar{x}_{k}-\bar{x}_{j}\right) p_{j}}{K\left(\bar{x}_{k}\right)}-\frac{\mathrm{a}_{j} a\left(\bar{x}_{i}-\bar{x}_{j}\right) p_{j}}{K\left(\bar{x}_{i}\right)}+O\left(e^{2}\right) .
\end{aligned}
$$

Inserting this into eq. (7) gives the dynamics of the frequency $p_{i}$ of morph $i$, in leading order of $e$,

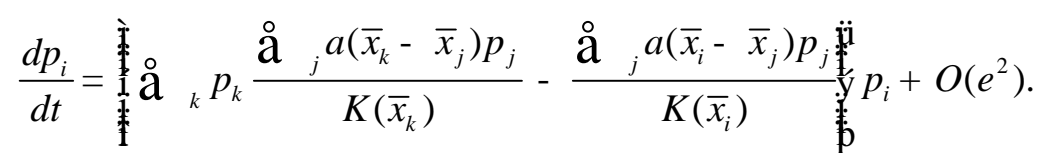

Since this result has the form of a replicator equation, $\frac{d}{d t} p_{i}=\left(\bar{w}_{i}-\bar{w}\right) p_{i}$, with $\bar{w}=\stackrel{\mathrm{a}}{ }{ }_{i} \bar{w}_{i} p_{i}$, we can interpret $b_{i j}=a\left(\bar{x}_{i}-\bar{x}_{j}\right) / K\left(\bar{x}_{i}\right)$ as the effective interaction coefficient describing the effect of morph $j$ on the frequency of morph $i$.

\subsubsection{Equilibria of morph frequencies}

Morph means $\bar{x}=\left(\bar{x}_{1}, \mathrm{~K}, \bar{x}_{n}\right)^{\mathrm{T}}$ usually change much more slowly than morph frequencies $p=\left(p_{1}, \mathrm{~K}, p_{n}\right)^{\mathrm{T}}$. This is because the ecological dynamics in eq. (18), which have rates of order $\varepsilon^{0}$, are much faster than the evolutionary dynamics in eq. (24) below, which have rates of order $\varepsilon^{2}$, as long as the within-morph variances $V_{i}=O\left(\varepsilon^{2}\right)$ are sufficiently small. Accordingly, morph means stay almost constant while morph frequencies approach a quasi-equilibrium $p(\bar{x})$ with $\AA^{\circ}{ }_{j} b_{i j} p_{j}(\bar{x})=\AA_{j}{ }_{j k} b_{k j} p_{j}(\bar{x}) p_{k}(\bar{x})$ for all $i=1, \mathrm{~K}, n$. These conditions can be spelled out as

$$
\frac{1}{K\left(\bar{x}_{i}\right)} \mathrm{a}_{j} a\left(\bar{x}_{i}-\bar{x}_{j}\right) p_{j}(\bar{x})=\AA_{j k} \frac{1}{K\left(\bar{x}_{k}\right)} a\left(\bar{x}_{k}-\bar{x}_{j}\right) p_{j}(\bar{x}) p_{k}(\bar{x}),
$$

or rewritten in matrix form as

$$
(V A) p(\bar{x})=c u \text { with } c=p(\bar{x})^{\mathrm{T}}(V A) p(\bar{x}) \text {, }
$$

where $A=\left(A_{i j}\right)=\left(a\left(\bar{x}_{i}-\bar{x}_{j}\right)\right)$ is the interaction matrix, $V=\operatorname{diag}\left(1 / K\left(\bar{x}_{1}\right), \mathrm{K}, 1 / K\left(\bar{x}_{n}\right)\right)$, and $u=(1,1, \mathrm{~K}, 1)^{\mathrm{T}}$. With $\bar{K}=\left(K\left(\bar{x}_{1}\right), \mathrm{K}, K\left(\bar{x}_{n}\right)\right)^{\mathrm{T}}=V^{-1} u$, we thus obtain the quasi-equilibrium frequencies of morphs with means $\bar{x}$,

$$
p(\bar{x})=c A^{-1} \bar{K}=\frac{A^{-1} \bar{K}}{\mathrm{a}_{j}\left(A^{-1} \bar{K}\right)_{j}} .
$$

\subsubsection{Demographic stability}

Eq. (18) shows that the dynamics of morph frequencies are locally asymptotically stable around $p(\bar{x})$, if the eigenvalues of the Jacobian $D=\left(D_{i j}\right)$ with elements 


$$
\begin{aligned}
& D_{i j}=\left.\frac{\prod}{\llbracket p_{j}} p_{i}\left\{p^{\mathrm{T}}(V A) p-(V A p)_{i}\right\}\right|_{p=p(\bar{x})}
\end{aligned}
$$

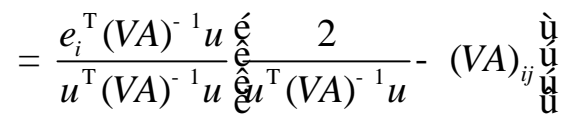

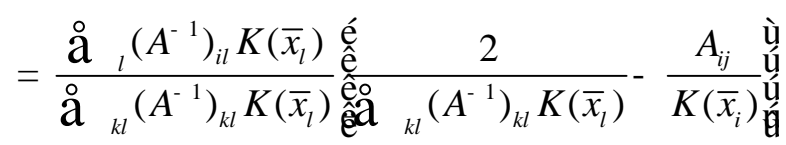

all have negative real parts, where $e_{i}$ is the unit vector along the $i$ th coordinate. Hence, this is the condition for the demographic stability of a population comprised of morphs with means $\bar{x}=\left(\bar{x}_{1}, \mathrm{~K}, \bar{x}_{n}\right)^{\mathrm{T}}$ and quasi-equilibrium frequencies $p(\bar{x})=\left(p_{1}(\bar{x}), \mathrm{K}, p_{n}(\bar{x})\right)^{\mathrm{T}}$ according to eq. (21). If this condition is violated, at least one morph will go extinct before the population becomes demographically stable.

\subsubsection{Dynamics of morph means}

The mean character $\bar{x}_{i}=$ ò $x f_{i}(x) d x$ of morph $i$ changes according to

$$
\begin{aligned}
\frac{d \bar{x}_{i}}{d t} & =\text { ò } x \frac{d f_{i}(x)}{d t} d x=\text { ò }^{x}\left\{w(x)-\bar{w}_{i}\right\} f_{i}(x) d x \\
& =\text { ò } x_{i}\left\{w(x)-\bar{w}_{i}\right\} f_{i}(x) d x,
\end{aligned}
$$

where $x_{i}=x-\bar{x}_{i}$. Substituting eqs. (12)-(15) into the right-hand side of eq. (23) yields, in leading order of $e$,

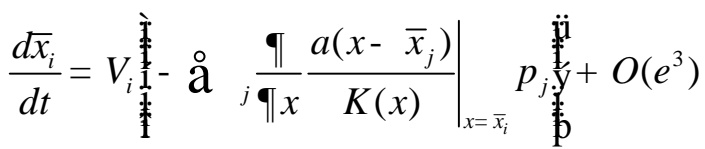

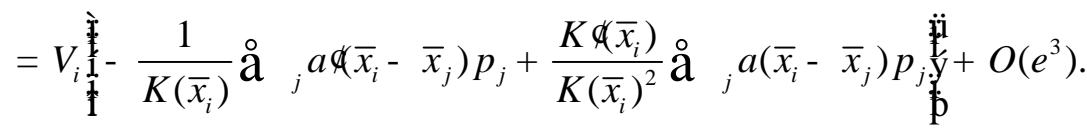

By noting that $w(x)=1-\stackrel{\circ}{{ }_{j}} p_{j} a\left(x-\bar{x}_{j}\right) / K(x)$, we see that this is equivalent to Wright's formula

$$
\frac{d \bar{x}_{i}}{d t}=\left.V_{i} \frac{\boldsymbol{\Phi} w(x)}{\mathbb{\prod} x}\right|_{x=\bar{x}_{i}},
$$

for the change in a character's mean. Thus, the mean of each morph evolves in the direction towards which its fitness increases, with the rate of this adaptation being proportional to the morph variance and to the steepness of the fitness gradient. The fitness gradient, given by the curly brace on the right-hand side of eq. (24), comprises two components. The first term drives morphs away from each other, while the second term pushes morphs towards the carrying capacity's maximum. 


\subsubsection{Equilibria of morph means}

It is clear from eq. (24) that the equilibrium morph means and their stability depend only on the means $\bar{x}_{i}$ and frequencies $p_{i}$, since the variances $V_{i}$ affect only the speed of convergence to, or divergence from, those equilibrium morph means. The equilibrium means and frequencies of morph $i$ then satisfy the following equations, in conjunction eq. (21),

$$
\stackrel{\circ}{\mathrm{a}}_{j} a\left(\bar{x}_{i}-\bar{x}_{j}\right) p_{j}=\frac{K \Phi\left(\bar{x}_{i}\right)}{K\left(\bar{x}_{i}\right)} \stackrel{\circ}{\mathrm{a}}{ }_{j} a\left(\bar{x}_{i}-\bar{x}_{j}\right) p_{j} .
$$

Defining the matrix $A^{\mathbb{\Phi}}=\left(A_{i j}^{\Phi}\right)=\left(a \Phi\left(\bar{x}_{i}-\bar{x}_{j}\right)\right)$ and the diagonal matrix $U=\operatorname{diag}\left(K \Phi\left(\bar{x}_{1}\right) / K\left(\bar{x}_{1}\right), \mathrm{K}, K \Phi\left(\bar{x}_{n}\right) / K\left(\bar{x}_{n}\right)\right)$, eq. (26) can be rewritten in matrix form as $A \Phi p=U A p$. Substituting for $p$ the equilibrium frequencies $p(\bar{x})=c A^{-1} \bar{K}$ derived in eq. (21), we obtain $A \AA^{-1} \bar{K}=U \bar{K}=\bar{K} \pitchfork$ with $\bar{K} \Phi=\left(K \Phi\left(\bar{x}_{1}\right), K, K \Phi\left(\bar{x}_{n}\right)\right)^{\mathrm{T}}$. Spelled out, this gives

$$
\stackrel{\circ}{\mathrm{a}}_{j k} a థ\left(\bar{x}_{i}-\bar{x}_{j}\right)\left(A^{-1}\right)_{j k} K\left(\bar{x}_{k}\right)=K \phi\left(\bar{x}_{i}\right)
$$

for $i=1, \mathrm{~K}, n$, which determines the equilibrium means $\bar{x}_{i}$ of each morph.

\subsubsection{Convergence stability}

To assess the stability of the equilibrium morph means $\left(\bar{x}_{1}, \bar{x}_{2}, \mathrm{~L}, \bar{x}_{n}\right)^{\mathrm{T}}$ under the dynamics in eq. (24), we investigate the corresponding Jacobian. The diagonal elements of this Jacobian are given by

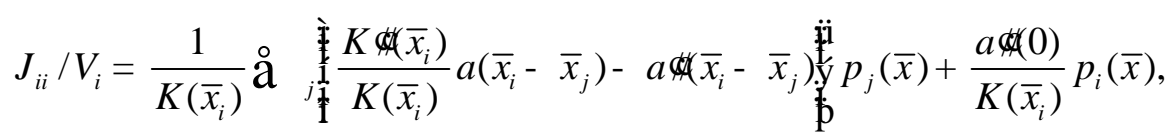

where we used eq. (26). Similarly, the off-diagonal elements of the Jacobian are given by

$$
J_{i j} / V_{i}=\frac{1}{K\left(\bar{x}_{i}\right)} a \Phi\left(\bar{x}_{i}-\bar{x}_{j}\right) p_{j}(\bar{x})-\frac{K \Phi\left(\bar{x}_{i}\right)}{K\left(\bar{x}_{i}\right)^{2}} a థ\left(\bar{x}_{i}-\bar{x}_{j}\right) p_{j}(\bar{x}) .
$$

It is interesting to compare the condition for the stability of the dynamics of morph means in eq. (24) with the condition for convergence stability (Eshel and Motro 1981; Eshel 1983). In general, a character value $x$ is said to be convergence stable if character values closer to $x$ can invade when the resident character value of the otherwise monomorphic morph is slightly displaced from $x$. To establish this link, we consider a resident population consisting of an atomic distribution composed of $n$ monomorphic peaks at character values $\bar{x}_{j}$ and with frequencies $p_{j}(\bar{x})$ for $j=1, \mathrm{~K}, n$, which can be represented as å ${ }_{j} p_{j}(\bar{x}) d\left(x-\bar{x}_{j}\right)$, where $d$ is Dirac's delta function. The invasion fitness $s_{y}(x)$ (Metz et al. 1992) of a variant character value $x$ in a population in which the resident character value $y$ of morph $i$ is slightly displaced from its equilibrium value $\bar{x}_{i}$, while the other morphs are at their equilibrium value $\bar{x}_{j}$, is then given by

$$
s_{y}(x)=-\frac{1}{K(x)} \stackrel{\mathrm{a}}{j^{1} i}{ }^{1} a\left(x-\bar{x}_{j}\right) p_{j}(\bar{x})-\frac{1}{K(x)} a(x-y) p_{i}(\bar{x}) .
$$


The condition for the convergence stability of the character value $\bar{x}_{i}$ is

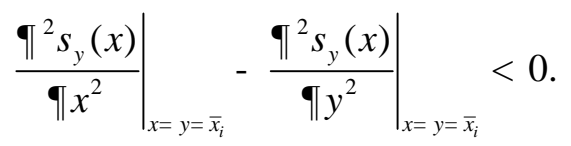

This is equivalent to the condition $J_{i i}<0$ for the asymptotic stability of the dynamics in eq. (24) in the special case that only a single morph mean $\bar{x}_{i}$ is displaced at a time. A more general result for a character distribution's stability against simultaneous perturbation in the positions of multiple morphs will be presented elsewhere (Sasaki and Dieckmann, in preparation).

\subsubsection{Dynamics of morph variances}

The variance $V_{i}=$ Ò $x_{i}^{2} f_{i}(x) d x$ of morph $i$ changes according to

$$
\frac{d V_{i}}{d t}=\grave{\mathbf{O}} x_{i}^{2} \frac{d f_{i}}{d t} d x=\grave{\mathbf{O}} x_{i}^{2}\left\{w(x)-\bar{w}_{i}\right\} f_{i}(x) d x \text {. }
$$

If the character distribution $f_{i}$ of each morph $i=1, \mathrm{~K}, n$ is symmetric around its mean $\bar{x}_{i}, f_{i}\left(\bar{x}_{i}+x_{i}\right)=f_{i}\left(\bar{x}_{i}-x_{i}\right)$, all odd moments of $f_{i}$ in terms of $x_{i}$ vanish. Using eqs. (12)-(15) then yields, in leading order of $e$,

$$
\frac{d V_{i}}{d t}=F_{i}\left\{Q_{i}-V_{i}^{2}\right\}+O\left(e^{5}\right),
$$

where $F_{i}=\frac{1}{2} \mathbb{q}^{2} w(x) /\left.\mathbb{q} x^{2}\right|_{x=\bar{x}_{i}}$ and $Q_{i}=E_{f_{i}}\left[x_{i}^{4}\right]=\mathrm{O} x_{i}^{4} f_{i}(x) d x$ is the fourth moment of the character distribution of morph $i$.

\subsubsection{Equilibria of morph variances}

Since $E_{f_{i}}\left[x_{i}^{4}\right]-V_{i}^{2}=E_{f_{i}}\left[\left(x_{i}^{2}-V_{i}\right)^{2}\right] \quad 3 \quad 0, Q_{i}-V_{i}^{2}$ is always positive, so the local asymptotic stability of the dynamics in eq. (33) is determined by the sign of

$$
\begin{aligned}
& F_{i}=-\left.\frac{1}{2} \stackrel{\circ}{a} \frac{\boldsymbol{I}^{2}}{\boldsymbol{q} x^{2}} \frac{a\left(x-\bar{x}_{j}\right)}{K(x)}\right|_{x=\bar{x}_{i}} p_{j}
\end{aligned}
$$

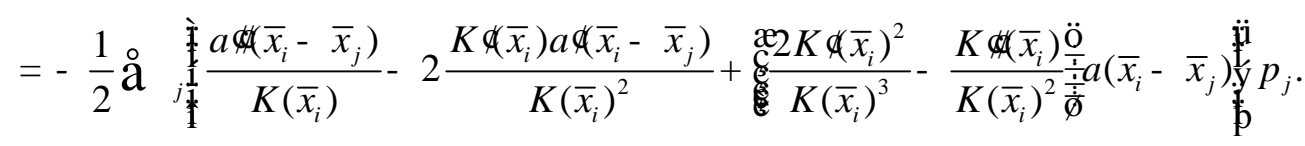

Consequently, $V_{i}$ increases if $F_{i}>0$ and decreases if $F_{i}<0$.

\subsubsection{Evolutionary stability}

When morph frequencies and means are at their equilibrium values, eq. (29) reduces to

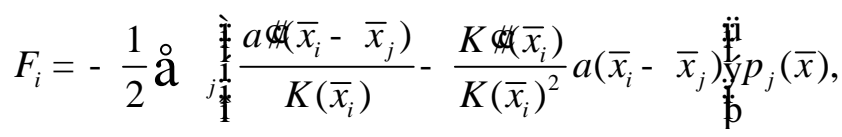

where we used eq. (26). Thus, the equilibrium $V_{1}=L=V_{n}=0$ of eq. (33) is locally asymptotically stable if all $F_{i}$ are negative. It is therefore possible that all morph means converge to a stable equilibrium, while one or more of the morph variances are unstable and, 
according to eq. (33), diverge to infinity. This happens for morph $i$, if $F_{i}$ is positive at the stable equilibrium of the combined dynamics of morph means and frequencies. If, in contrast, $F_{i}$ is negative, the variance of morph $i$ gradually vanishes.

It is interesting to compare the condition for the stability of the dynamics of morph variances in eq. (33) with the condition for local evolutionary stability (Maynard Smith 1982; Brown and Vincent 1987). In general, a character value $x$ is said to be locally evolutionarily stable if character values close to $x$ cannot invade an otherwise monomorphic morph with resident character value $x$. To establish this link, we again consider a resident population consisting of an atomic distribution composed of $n$ monomorphic peaks at character values $\bar{x}_{j}$ and with frequencies $p_{j}(\bar{x})$ for $j=1, \mathrm{~K}, n$, resulting in the invasion fitness in eq. (30). The condition for the local evolutionary stability of the character value $\bar{x}_{i}$ is

$$
\left.\frac{\coprod^{2} s_{y}(x)}{\mathbb{\prod} x^{2}}\right|_{x=y=\bar{x}_{i}}<0 .
$$

Inserting eq. (30), this yields $2 F_{i}<0$, so that all morph means are locally evolutionarily stable if and only if the corresponding morph variances converge to zero. Since $F_{i}$ is the second derivative of fitness at the mean of morph $i, F_{i}>0$ implies a fitness minimum and, consequently, that selection on this morph is disruptive.

\subsubsection{Moment closure}

Although the stability of the dynamics of morph variances in eq. (33) does not depend on the fourth moments $Q_{i}$ of the character distributions $f_{i}$ of morphs $i$, we need to specify these fourth moments so as to close the hierarchy of moment dynamics that jointly describes changes in morph frequencies, means, and variances according to eqs. (18), (24), and (33)-(34).

Approximating $f_{i}$ by a Gaussian distribution with mean $\bar{x}_{i}$ and variance $V_{i}$ yields $E_{f_{i}}\left[x_{i}^{3}\right]=0$ and $Q_{i}=E_{f_{i}}\left[x_{i}^{4}\right]=3 V_{i}^{2}$. Substituting this into eq. (33) gives

$$
\frac{d V_{i}}{d t}=2 F_{i} V_{i}^{2}
$$

If, by contrast, the character variations within each morph around its mean obey the house-of-cards model of mutation (Turelli 1984), then $V_{i}=E_{f_{i}}\left[x_{i}^{2}\right]=c_{2} m, E_{f_{i}}\left[x_{i}^{3}\right]=0$, and $Q_{i}=E_{f_{i}}\left[x_{i}^{4}\right]=c_{4} m$ where $m$ is the mutation rate and $c_{2}$ and $c_{2}$ are constants determined by the strength of stabilizing selection around $\bar{x}_{i}$. Substituting $Q_{i}=\left(c_{4} / c_{2}\right) V_{i}$ into eq. (33) gives, in leading order of $e$,

$$
\frac{d V_{i}}{d t}=\left(c_{4} / c_{2}\right) F_{i} V_{i}
$$


where we dropped the term $-F_{i} V_{i}^{2}$, since it is of order $O\left(e^{4}\right)$. The local asymptotic stability of the variance dynamics in eq. (38) again depends only on the sign of $F_{i}$, and thus on the fitness curvature at $\bar{x}_{i}$.

\subsubsection{Time to evolutionary branching}

For the Gaussian closure, eq. (37) determines not only the evolutionary stability of equilibrium morph means, but also the time a morph's character distribution needs to undergo evolutionary branching. If the fitness landscape $w(x)$ is locally disruptive at $\bar{x}_{i}$, implying $F_{i}>0$, the variance $V_{i}(t)=\left[2 F_{i}\left(t_{c}-t\right)\right]^{-1}$ diverges to infinity from an initial value $V_{i}(0)$ within a finite time $t_{\mathrm{c}}$,

$$
t_{\mathrm{c}}=\left[2 F_{i} V_{i}(0)\right]^{-1} \text {. }
$$

Obviously, the assumption of small morph variances, which is necessary for the oligomorphic dynamics to provide a good approximation, fails before a morph variance approaches infinity. The duration $t_{\mathrm{c}}$ nevertheless provides a useful approximation of the time to evolutionary branching required by a morph that experiences disruptive selection of strength $F_{i}$ (Fig. 1).

For the house-of-cards closure, the transient dynamics to evolutionary branching is more gradual. The variance diverges exponentially with a rate that is proportional to the strength of disruptive selection. Hence, the characteristic time $t_{\mathrm{c}}$ to evolutionary branching,

$$
t_{\mathrm{c}}=\left[\left(c_{4} / c_{2}\right) F_{i}\right]^{-1}
$$

is again inversely proportional to $F_{i}$. Despite this similarity, the exponential mode of divergence described by eq. (38) is in qualitative contrast to the explosive divergence after a long period of near-stasis that results for the Gaussian closure.

\subsubsection{Effects of mutation on morph variances}

The variance of quantitative characters subject to stabilizing selection can be maintained by mutation-selection balance: the character diversity that gets depleted by purifying selection is then restored by the generation of variation through mutation (Bulmer 1972; Lande 1975, and references therein; Barton and Turelli 1991). Denoting the rate of mutation by $m$ and assuming that mutational effects on character values are random with variance $\mathrm{m}^{2}$ (corresponding to the constant-variance model or random-walk model of quantitative genetics theory), the dynamics of morph variances in the oligomorphic model is modified as

$$
\frac{d V_{i}}{d t}=F_{i}\left\{Q_{i}-V_{i}^{2}\right\}+m m^{2}
$$

where $F_{i}$ again measures the strength of disruptive selection around $\bar{x}_{i}$ according to eq. (34) in general and to eq. (35) for the case that the $\bar{x}_{i}$ have attained a convergence stable equilibrium. 
For $F_{i}>0, V_{i}$ diverges to infinity as in the absence of mutations, but for $F_{i}<0$, equilibrium morph variances $V_{i}>0$ are stabilized. For the Gaussian closure, these are given by

$$
V_{i}=\sqrt{\frac{m m^{2}}{2 F_{i}}},
$$

and for the house-of-cards closure by

$$
V_{i}=\frac{m m^{2}}{\left(c_{4} / c_{2}\right) F_{i}}
$$

\section{Applications of oligomorphic dynamics}

We now use the oligomorphic approximation derived above to understand in detail the dynamics of, and the morph patterns resulting from, evolutionary branching in the resource-competition model. The dynamical equations that we integrate numerically describe the frequencies, means, and variances of morphs as given by eqs. (18), (24), and (33)-(34), which we assemble here for ease of reference,

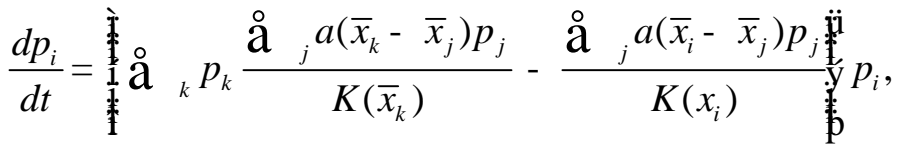

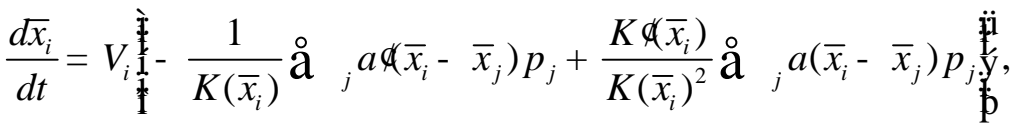

$$
\begin{aligned}
& \frac{d V_{i}}{d t}=-\frac{1}{2}\left\{Q_{i}-V_{i}^{2}\right\} \text { å } \underset{j, \frac{1}{1}}{\frac{1}{1}} \frac{a \Phi\left(\bar{x}_{i}-\bar{x}_{j}\right)}{K\left(\bar{x}_{i}\right)}-2 \frac{K\left(\bar{x}_{i}\right) a \Phi\left(\bar{x}_{i}-\bar{x}_{j}\right)}{K\left(\bar{x}_{i}\right)^{2}}
\end{aligned}
$$

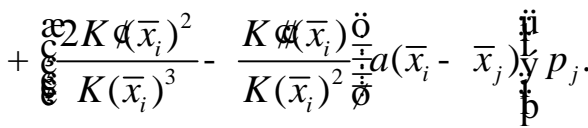

While the numerical analysis of eqs. (44) starts with a fixed number $n$ of morphs, the subsequent eco-evolutionary dynamics may effectively reduce this number. This may occur because morph frequencies become negligible or because morph means become indistinguishable. For example, starting with five morphs when the equilibrium is dimorphic, three morphs will subsequently be lost in such a manner.

\subsection{Special case allowing continuous morph distributions}

In the special case in which the competition kernel $a$ and the carrying-capacity function $K$ are both Gaussian, $a(x)=\exp \left(-\frac{1}{2} x^{2} / s^{2}\right)$ and $K(x)=\exp \left(-\frac{1}{2} x^{2} / w^{2}\right)$, and the former is narrower than the latter, $s<w$, the character distribution in eqs. (44) converges, through incessant evolutionary branching, to a continuum of infinitesimally spaced morphs. Accordingly, the number of morphs that can be packed along the niche character $x$ is unlimited (Ma- 
cArthur 1970; Roughgarden 1972; May 1973, 1974; Slatkin and Lande 1976; Bull 1987). However, many deviations from this special case, e.g., by choosing a competition kernel or carrying-capacity function that are not Gaussian, result in atomic distributions, i.e., in the coexistence of discrete (that is, finitely spaced) morphs (Sasaki and Ellner 1995; Sasaki 1997; Gyllenberg and Meszéna 2005; Szabó and Meszéna 2006; Pigolotti et al. 2007, 2009; Leimar et al. 2008; Fort et al. 2009).

Integrating eqs. (44) with the house-of-card closure in eq. (38) shows that for both $n=2$ (Figure 2A, B) and $n=5$ (Figure 2C, D) morph means become displaced from their initial values and relative to each other, while morph variances increase without limit, indicating that neither two nor five morphs are enough to evolutionarily stabilize the population. It turns out that this conclusion is independent of $n$. Below we show how this degeneracy is overcome for $s>w$ or by varying the kurtoses of the competition kernel or the carrying-capacity function.

\subsection{Single-morph dynamics}

When there is only one morph in the population $(n=1)$, its mean and variance change according to

$$
\begin{aligned}
& \frac{d \bar{x}}{d t}=V \frac{K \Phi(\bar{x})}{K(\bar{x})},
\end{aligned}
$$

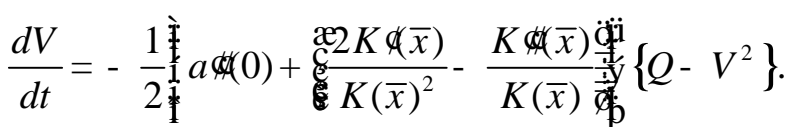

The mean $\bar{x}$ of a single morph thus always converges to the carrying capacity's maximum at $x=0$.

At this convergence stable equilibrium for the mean, the variance dynamics reduce to

$$
\frac{d V}{d t}=\frac{1}{2}\{K \phi(0)-a \notin(0)\}\left\{Q-V^{2}\right\} \text {. }
$$

Thus, the convergence stable equilibrium $\bar{x}$ is also evolutionarily stable, and the morph variance $V$ hence remains finite, if and only if

$$
a \notin(0)<K \phi(0) \text {. }
$$

We can interpret this condition by concluding that evolutionary stability requires the width $1 /|a(0)|$ of the competition kernel, as described by its peak curvature, to exceed the corresponding width $1 /|K \propto(0)|$ of the resource distribution. This is equivalent to $s>w$, a condition that was already derived by Roughgarden (1972). If, on the other hand, this condition is violated, the morph variance $V$ diverges to infinity. This implies that $x=0$ is convergence stable, as the morph mean approaches $x=0$, but not evolutionarily stable, as the variance around $x=0$ increases without limit. The character value $x=0$ is therefore an evolutionary branching point when inequality (47) is violated. 


\subsection{Two-morph dynamics}

\subsubsection{Frequency dynamics and limiting similarity}

When there are only two morphs in the population $(n=2)$, the frequency of one of them, $p_{1}=p$ (with $p_{2}=1-p$ ), changes according to

$$
\frac{d p}{d t}=s\left(p_{c}-p\right) p(1-p)
$$

with

$$
s=\frac{\left(K_{1}+K_{2}\right)(1-a(\mathrm{D}))}{K_{1} K_{2}} \text { and } p_{\mathrm{c}}=\frac{K_{1}-K_{2} a(\mathrm{D})}{\left(K_{1}+K_{2}\right)(1-a(\mathrm{D}))} \text {, }
$$

where $K_{i}=K\left(\bar{x}_{i}\right)$ is the carrying capacity of morph $i=1,2$ and $a(\mathrm{D})$ with $\mathrm{D}=\bar{x}_{1}-\bar{x}_{2}$ is the competition coefficient between morph 1 and morph 2, which decreases as the character displacement $\mathrm{D}$ increases. Note that both $K_{i}$ and $a(\mathrm{D})$ are time-dependent, because $\bar{x}_{1}$ and $\bar{x}_{2}$ change with time, at a speed that is slow compared with the speed of the frequency dynamics in eq. (48). For a given pair $\bar{x}_{1}$ and $\bar{x}_{2}$, the frequency $p$ is attracted towards the equilibrium value $p_{c}$.

Eqs. (48) and (49) imply that if the two morphs are sufficiently separated from each other (|D|? $s$, where $s$ is the standard deviation of the competition kernel $a$ ), then $a(\mathrm{D})=1$ and the two morphs are subject to strong balancing selection with equilibrium frequency $p_{c}$. If, in contrast, the two morphs are sufficiently close to each other $(|\mathrm{D}|=s)$, then $a(\mathrm{D})$ " 1 and the balancing selection is weak. If the two morphs have the same carrying capacity $\left(K_{1}=K_{2}\right)$, which occurs when the dimorphism is symmetric, $\bar{x}_{1}=-\bar{x}_{2}$, the equilibrium frequency $p_{\text {c }}$ converges to $1 / 2$. If the ratio $K_{1} / K_{2}$ between the carrying capacity of morph 1 and that of morph 2 is smaller than the competition coefficient, $K_{1} / K_{2}<a(\mathrm{D})$, morph 1 goes extinct. Analogously, for $K_{2} / K_{1}<a(\mathrm{D})$, morph 2 goes extinct. These results for the two-morph frequency dynamics are fully in line with conventional limiting-similarity theory (May 1974).

\subsubsection{Branching patterns and effects of kurtosis}

An interesting application of oligomorphic dynamics as developed above is to study the bifurcations that occur when inequality (47) is violated, so that evolutionary branching can happen. Below we show that the resultant branching patterns sensitively depend on the kurtoses of the competition kernel $a$ and of the carrying-capacity function $K$. We therefore consider these functions to be symmetric and allow them to be either platykurtic or leptokurtic. Under these conditions, an initially symmetric dimorphism resulting from the evolutionary branching of a single morph at $x=0$ remains symmetric: $p_{1}(t)=p_{2}(t)=1 / 2$, $\bar{x}_{1}(t)=-\bar{x}_{2}(t)$, and $V_{1}(t)=V_{2}(t)$ for all $t$. Moreover, numerical investigations of the two-morph dynamics, eqs. (44) with $n=2$, demonstrate that for an initially asymmetric di- 
morphism, with $\bar{x}_{1}(0)^{1}-\bar{x}_{2}(0)$, in which $\bar{x}_{1}(0)$ and $\bar{x}_{2}(0)$ are both close to 0 , the symmetry between the two morphs is rapidly established long before their means equilibrate.

Defining $\bar{x}=\bar{x}_{1}=-\bar{x}_{2}$ and $V=V_{1}=V_{2}$, substituting these into the mean and variance dynamics in eqs. (44), and doubly expanding the resulting equations in Taylor series around $\bar{x}=0$ and $x=x-\bar{x}=x$ yields

$$
\frac{d \bar{x}}{d t}=\{K \Phi(0)-a \notin(0)\} \bar{x} V+\frac{1}{6}\left\{9 a \phi(0) K \phi(0)-6 K \phi(0)^{2}-4 a d(0)+K\right.
$$

and

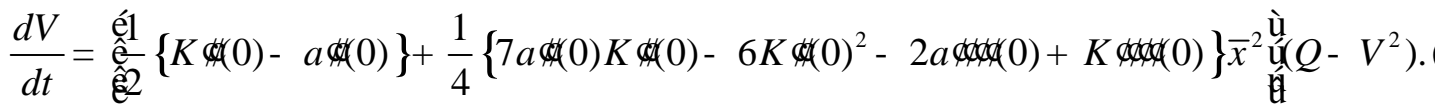

The parameter $d=K \Phi(0)-a \Phi(0)$ measures the net disruptiveness of fitness at $\bar{x}=0$, so that $d=0$ corresponds to the bifurcation point for primary evolutionary branching. Using the order estimate $\bar{x}=O(\sqrt{d})$, we obtain in leading order of $d$

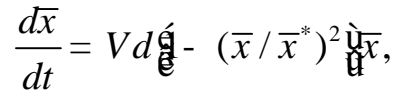

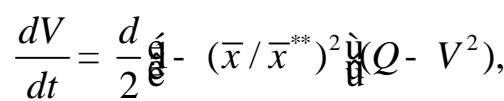

with

$$
\bar{x}^{*}=\frac{\sqrt{d}}{|a+(0)| \sqrt{1-2 g_{a} / 3+g_{K} / 6}} \text { and } \bar{x}^{* *}=\frac{\sqrt{d}}{|a+(0)| \sqrt{1-g_{a}+g_{K} / 2}} \text {, }
$$

where $g_{a}=3-a$ a $/ a \phi(0)^{2}$ and $g_{K}=3-K \phi(0) / K \phi(0)^{2}$ measure the excess kurtoses of the competition kernel and carrying-capacity functions, respectively (i.e., the deviations of the fourth moments of $a$ and $K$ from their expectations $3 a \leftarrow(0)^{2}$ and $3 K \Phi(0)^{2}$ in the Gaussian case). For a net disruptiveness of $d=K \Phi(0)-a \notin(0)<0$, both the character displacement $\mathrm{D}=2 \bar{x}$ between the two morphs and the variance $V$ of both morphs converge to zero, indicating that the population converges to monomorphism at $x=0$.

For $d>0$, this monomorphism is unstable. There are then two qualitatively different behaviors, depending on the kurtoses of the competition kernel and carrying-capacity function. If the carrying-capacity function is more platykurtic than the competition kernel $\left(g_{K}>g_{a}\right)$, then $\bar{x}$ increases towards $\bar{x}^{*}$. As character displacement increases, the morph variances first increase and then decrease towards zero once $\bar{x}$ exceeds $\bar{x}^{* *}$ (Figure 3A-C). Thus, the population converges to an atomic distribution at $\pm \bar{x}^{*}$. If the competition kernel is more platykurtic than the carrying-capacity function $\left(g_{a}>g_{K}\right)$, then $\bar{x}^{* *}>\bar{x}^{*}$, which implies that the morph variances keep increasing even after the morph means have reached their equilibrium (Figure 3D-F). The two morph variances therefore increase without limit, indicating that the dimorphism $\pm \bar{x}^{*}$ is not evolutionarily stable. In this case, a trimorphism, rather than a dimorphism, is the successor of the initial monomorphism, as will be illustrated in more detail 
below. Figure 3C and 3F depicts trajectories $(\bar{x}, V)$, as well as the isoclines $d \bar{x} / d t=0$ and $d V / d t=0$, for $g_{K}>g_{a}$ (Figure 3C) and $g_{a}>g_{K}$ (Figure 3F).

Figure 4 shows results of the numerical analysis of the corresponding two-morph and three-morph dynamics. As a robustness check, we consider an initially asymmetric dimorphism, and verify that symmetry is nonetheless subsequently established. The carrying-capacity function is platykurtic, indeed purely quartic, $K(x)=\exp \left(-\frac{1}{12} x^{4} / h^{4}\right)$, and the competition kernel is Gaussian, $a(x)=\exp \left(-\frac{1}{2} x^{2} / s^{2}\right)$. For these specific functions, a pairwise invasibility analysis of the symmetric dimorphism $\pm \bar{x}^{*}$ reveals that for $h / s<1.16$ this dimorphism is evolutionarily stable, while for $h / s>1.16$ it is destabilized (Figure 5).

\subsection{Effects of mutation on evolutionary branching}

We now examine how mutations affect the condition for evolutionary branching. For this, we consider a Gaussian competition kernel $a$ and a resource distribution $K$ that can be either Gaussian or platykurtic,

$$
a(x)=\exp \left(-\frac{1}{2} x^{2}\right) \text { and } K(x)=\exp \left(-\frac{1}{2} x^{2} / w^{2}-g x^{4}\right) .
$$

The character $x$ is scaled so that the standard deviation of the competition kernel equals 1 , $w$ measures the standard deviation of the resource distribution, and $g^{3} 0$ determines the degree of platykurtosis of the resource distribution. According to inequality (47), the threshold for evolutionary branching in the absence of mutation is given by $w=1$. In the special, and highly structurally unstable, case that both competition kernel and resource distribution are Gaussian ( $g=0)$, and when the resource distribution is wider than the competition kernel $(w>1)$, a continuous distribution with variance $w^{2}-1$ is stable (MacArthur and Levins 1967; MacArthur 1969, 1970; Roughgarden 1972; May 1973, 1974; Slatkin and Lande 1976; Bull 1987). If, in contrast, the resource distribution is just slightly platykurtic $(g>0)$, the dynamic outcome abruptly changes into an evolutionarily stable dimorphism (Sasaki and Ellner 1995; Ellner and Sasaki 1996; Sasaki 1997).

If recurrent mutations generate variance, atomic character distributions cannot remain atomic; instead, each morph must feature narrow blurs around its peaks. So far, however, there has been little study of how mutations change the bifurcations associated with evolutionary branching, or the character distributions that from evolutionary branching. It is also interesting to ask how adding mutations affects the structurally unstable continuous distributions expected for the combination of Gaussian competition kernels with Gaussian resource distributions. In this section, we apply oligomorphic dynamics to answer these three questions. For this purpose, we assume that, owing to mutations, an offspring's character deviates from that of its parent with rate $m$ and variance $m^{2}$. 


\subsubsection{Analytical results}

Analyzing the dynamics of a single morph, we focus on cases in which the net disruptiveness $d=1-w^{-2}>0$ is close to the bifurcation point $d=0$, and expand, up to sixth order in the character deviation $x=x-\bar{x}$, the selection component $(d V / d t)_{\text {sel }}$ of the variance dynamics. As shown in Appendix A, this gives

$$
\underset{\varepsilon}{\not d t} \frac{\ddot{0}}{\dot{\bar{\Phi}}_{\text {sel }}}=\frac{1}{2} d\left(Q-V^{2}\right)-\frac{1}{2}\left(Q-V^{2}\right) V+g(H-V Q)
$$

where $V=$ ò $^{2} f(x) d x, Q=\mathbf{O}^{x^{4}} f(x) d x$, and $H=$ ò $^{x^{6}} f(x) d x$. Combining this with the mutation component

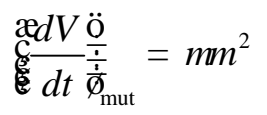

of the variance dynamics, we obtain the total rate of variance change as $d V / d t=$ $(d V / d t)_{\text {sel }}+(d V / d t)_{\text {mut }}$.

To derive from this a rough estimate of the equilibrium morph variance, we can assume that the character distribution is approximately Gaussian, so that $Q=3 V^{2}$ and $H=15 V^{3}$, which gives

$$
\frac{d V}{d t}=d V^{2}-(1+12 g) V^{3}+m m^{2} .
$$

Setting the right-hand side to 0 , we obtain the approximate equilibrium morph variance $V$ as an implicit function of the bifurcation parameters $d$ or $w=(1-d)^{-1 / 2} » 1+d / 2$. Figure 6 compares this with the results of numerical analyses.

As shown by the numerical analyses, mutations postpone the bifurcation towards multimodality in the character distribution that results from increasing the strength of disruptive selection. We examine how far mutations shift this bifurcation point, by assuming small deviations of characters from the mean of an approximately Gaussian character distribution. For a morph variance $V$, the two leading terms for the second derivative $w \mathbb{( 0 )}$ of fitness at the morph mean $\bar{x}=0$ are then given by

$$
w^{\mathbb{C}}(0)=d-V .
$$

Thus, if $d=1-w^{-2}$ exceeds the equilibrium morph variance $V$ defined by eq. (57), the fitness landscape is disruptive at $\bar{x}=0$. The bifurcation point $d$ at which this occurs is therefore obtained by substituting $V=d$ into eq. (57), setting its right-hand side to 0 , which yields

$$
-12 g d^{3}+m m^{2}=0 .
$$

This means that mutations shift the bifurcation point from $d=0$ to

$$
d=\sqrt[3]{\frac{m m^{2}}{12 g}},
$$


or equivalently, from $w=1$ to

$$
w=1+\frac{1}{2} \sqrt[3]{\frac{m m^{2}}{12 g}} .
$$

Consequently, for conditions close to $d=0$ and $g=0$, an arbitrary amount of mutation-induced variance $\mathrm{mm}^{2}$ prevents evolutionary branching. The special case of Gaussian competition kernels and resource distributions (which implies a dynamically stable, but structurally unstable, equilibrium character distribution of Gaussian shape), then loses its pathological nature (Sasaki and Ellner 1995; Sasaki 1997; Gyllenberg and Meszéna 2005; Pigolotti et al. 2007, 2009) and instead results in a structurally stable evolutionary outcome featuring a single evolutionarily stable morph.

\subsubsection{Numerical results}

Figure 6A shows how $w$ affects the equilibrium character distribution $f(x)$. The distribution stays unimodal for $d<0$ or $w<1$, in accordance with the predicted bifurcation points without mutation. For $g=0.05$ and $\mathrm{mm}^{2}=3.2 \times 10^{-4}$, the predicted bifurcation points with mutation are $d=\left(\mathrm{mm}^{2} / 12 \mathrm{~g}\right)^{1 / 3}=0.08196$ or $w=1.03976$. This well approximates the threshold at which the fitness landscape becomes bimodal (dashed line in Figure 6B). The equilibrium character distribution stays unimodal for even larger values of $w$ (Figure 6A), after the fitness landscape becomes bimodal (Figure 6B), with an increasing platykurtosis in the single morph compensating for the increasing disruptiveness, up to about $w=1.136$ (Figure 6D).

Figure 6B shows the equilibrium morph variance $V$ as a function of $w$. The variance gradually increases as the bifurcation parameter $w$ is raised. The numerical results (dotted line) are in good agreement with the approximate analytical results (continuous line), which are derived for small $d=1-w^{-2}$ and obtained as the root of the cubic equation that results from setting to 0 the right-hand side of eq. (57).

Figure $6 \mathrm{D}$ shows the equilibrium morph kurtosis $E_{f}\left[x^{4}\right] /\left(3 V^{2}\right)$ as a function of $w$. The kurtosis gradually decreases from 1 (for a mesokurtic distribution) as the bifurcation parameter $w$ is raised. For $w$ between 1 and 1.136, the equilibrium character distribution remains unimodal, but becomes increasingly platykurtic (Figure 6A). Instead of splitting the equilibrium character distribution and creating a dimorphism, disruptive selection is compensated by mutation, becoming absorbed in the platykurtosis of an evolutionarily stable morph. If $w$ is further increased, disruptive selection overcomes this mutation-induced morph cohesion, so that the equilibrium character distribution starts to become bimodal (Figure 6A). 


\section{Discussion}

Here we have derived oligomorphic dynamics as a new theoretical framework for examining the joint ecological and evolutionary dynamics of populations with multiple interacting morphs. Building on, and integrating, salient aspects from a wide range of preeminent preceding work (including Lande 1976, 1979, 1981, 1982; Roughgarden 1972, 1976; Bulmer 1974; Slatkin 1980; Iwasa et al. 1991; Abrams et al. 1993; Vincent et al. 1993), our approach helps moving beyond a focus on unimodal character distributions, often taken in models of quantitative genetics theory, and on negligible within-morph variance, often taken in models of adaptive dynamics theory. Through a double Taylor expansion of interaction coefficients and fitness landscapes around the means of all morphs existing in a population, we have derived the approximate dynamics of morph frequencies, means, and variances.

More in particular, we have shown how oligomorphic dynamics can help investigate processes of adaptive diversification driven by frequency-dependent disruptive selection. For this purpose, we have (1) shown how to interpret conditions for demographic stability, convergence stability, and evolutionary stability in terms of the moments of oligomorphic dynamics, (2) presented alternative moment closures suitable for oligomorphic dynamics, (3) derived approximations for assessing the waiting time until evolutionary branching, and (4) analyzed the effects of mutation on equilibrium morph variances. In addition, for a classical model of resource competition we have (5) elucidated the structural instability of continuous character distributions, (6) obtained threshold conditions for primary and secondary evolutionary branching, and (7) derived corrections for describing the effects of mutation on evolutionary branching.

There is a great variety of aspects that need to be considered when trying to understand processes of adaptive speciation in ways that do justice to the complexity of the corresponding natural systems (e.g., Dieckmann and Doebeli 2005). Models based on game-theoretical and phenotypic dynamics have been used to investigate complexities in the ecological underpinnings of speciation, whereas models based on population genetics or quantitative genetics have helped analyze complexities in the genetic underpinnings of speciation (see, e.g., Dieckmann et al. 2004 for reviews). Oligomorphic dynamics contribute to bridging between these approaches, by extending the multi-morph dynamics of adaptive dynamics theory with analyses of the effects of morph variance and of the effects of mutation, while extending the single-morph dynamics of quantitative genetics theory with analyses of evolutionary branching and of morph interactions.

In the spirit of such bridge building, we have investigated how mutations affect the bifurcation structure and equilibrium character distribution in processes of adaptive speciation. It turns out that mutations have a large effect on the threshold condition for the relative net disruptiveness of selection (defined as the difference between the strength of disruptive selection and the strength of stabilizing selection, divided by the strength of disruptive selection). 
Specifically, eq. (61) shows how mutations shift the threshold for this relative net disruptiveness away from the value of 1 that applies in the absence of mutation. Since the resultant deviation is proportional to the cubic root of the mutation variance, even a small mutation variance can significantly shift the disruptiveness necessary for adaptive speciation.

Earlier theoretical studies have investigated the time required for a population to shift evolutionarily from one local peak of its fitness landscape to another. These studies had highlighted three factors determining the pace of such a transition: the fitness difference between the peaks, the depth of the valley separating them, and the evolving population's effective size (Lande 1985, 1986; Newman et al. 1985; see also Whitlock 1995, 1997). While these earlier studies dealt with shifts between preexisting fixed fitness peaks, here we have answered the related but different question as to the time required until an initially unimodal character distribution splits into two distinct morphs under the influence of frequency-dependent disruptive selection. For asexually reproducing species, this characterizes the waiting time until adaptive speciation. We have found that this waiting time is inversely proportional to the strength of disruptive selection, as measured by the curvature of the fitness landscape at the evolutionary branching point. Oligomorphic dynamics can be used to estimate this curvature.

Analyses based on oligomorphic dynamics also shed light on the fundamental structural instability of continuous distributions of species under combinations of Gaussian competition kernels and Gaussian resource distributions assumed in seminal papers on species packing (MacArthur and Levins 1967; MacArthur 1969, 1970; Roughgarden 1972; May 1974) and on the evolution of within-family variance in fluctuating environments (Slatkin and Lande 1976; Bull 1987; Sasaki and Ellner 1995; Ellner and Sasaki 1996; Sasaki 1997). As proved by Sasaki and Ellner (1995) and Sasaki (1997), even the slightest deviation from the non-generic assumption of mesokurtic functions destroys the build-up of a continuum of species (sometimes referred to as a "continuous ESS"). The condition for primary evolutionary branching we have derived here from oligomorphic dynamics with mutations, for a Gaussian competition kernel and a potentially platykurtic resource distribution, explains why evolutionary branching is obstructed in doubly Gaussian models with mutations. In lieu of evolutionary branching, the equilibrium character distribution merely broadens and its kurtosis increases, so that its bulk becomes flatter and its tails become thinner. Up to a point, such platykurtosis absorbs the frequency-dependent disruptive selection and thereby prevents evolutionary branching. A similar effect is likely to occur with regard to the stochastic fluctuations in morph means that arise from random drift in populations of finite size. Even though we cannot study such fluctuations using the deterministic framework developed here, our results suggest that, in the presence of residual disruptiveness, the distribution of these means over time will also be platykurtic. Therefore, this effect provides an additional mechanism for the effective absorption of disruptiveness through platykurtosis. 
When a quantitative character is subject to frequency-dependent selection that is strongest among individuals with similar character values, as happens for resource competition or for fluctuating selection with a shifting optimum, the character distribution that generically evolves is discrete, rather than continuous, in the sense that it consists of several distinctly separated morphs. The previously held expectation of unlimitedly tight (continuous) packing of species or character values (MacArthur 1970; May 1973, 1974; Roughgarden 1972; Slatkin and Lande 1976; Bull 1987) is based on structurally unstable models combining Gaussian competition with a Gaussian or uniform carrying capacity (Sasaki and Ellner 1995; Sasaki 1997; Gyllenberg and Meszéna 2005; Szabó and Meszéna 2006; Pigolotti et al. 2007, 2009; Leimar et al. 2008; Fort et al. 2009). The robust emergence of distinctly separated morphs in evolving distributions of quantitative characters underscores the importance of oligomorphic dynamics for understanding a wide range of evolutionary phenomena.

For example, conclusions similar to those drawn for species packing apply to models of character displacement. Slatkin's seminal character-displacement model (Slatkin 1980) considered a Gaussian competition kernel (with standard deviation $s_{a}$ ) in conjunction with a Gaussian carrying-capacity function (with standard deviation $s_{K}$ ) along a one-dimensional niche space. His analyses showed that a Gaussian character distribution with variance $s_{K}^{2}-s_{a}^{2}$ will evolve (Slatkin 1979) if disruptive selection dominates stabilizing selection $\left(s_{K}^{2}>s_{a}^{2}+s_{\mathrm{e}}^{2}\right.$, where $s_{\mathrm{e}}^{2}$ is the environmental variance). However, the structural instability of the doubly Gaussian model is responsible for the neutral stability of this continuous equilibrium character distribution in Slatkin's model. We suggest that oligomorphic dynamics as developed here provide a useful theoretical tool for analyzing character displacement, especially when considering non-mesokurtic interaction functions.

Our study leaves room for many important extensions. For example, to apply oligomorphic dynamics to more general and realistic models of adaptive speciation, it will be desirable to investigate the feasibility of incorporating more complex ecological aspects, such as assortative mating, as well as more complex genetic aspects, such as multi-locus inheritance, recombination, diploidy, and random drift.

\section{Acknowledgements}

A.S. thanks the Precursory Research for Embryonic Science and Technology (PRESTO) Program of the Japan Science and Technology Agency (JST) for financial support of this work, and acknowledges a Grant-in-Aid for Scientific Research from the Japan Society for the Promotion of Science (JSPS) and support from The Graduate University for Advanced Studies (Sokendai). U.D. gratefully acknowledges financial support by the European Commission, through the Marie Curie Research Training Network FishACE and the Specific Targeted Research Project FinE, funded under the European Community's Sixth Framework Program. U.D. received additional support by the European Science Foundation, the Austrian Science 
SASAKI AND DIECKMANN: OLIGOMORPHIC DYNAMICS

Fund, the Austrian Ministry of Science and Research, and the Vienna Science and Technology Fund. 


\section{Appendix A}

Here we derive the dynamics governing the variance of a unimodal character distribution $f(x)$, through a double Taylor expansion up to sixth order in the small deviation $x=x-\bar{x}=x$ of characters from the morph mean $\bar{x}=0$,

$$
\frac{d V}{d t}=\grave{\mathbf{O}} x^{2}(w(x)-\bar{w}) f(x) d x .
$$

In the expression for the fitness $w(x)$, we first expand the interaction coefficient $a(x-y)$ around $x$,

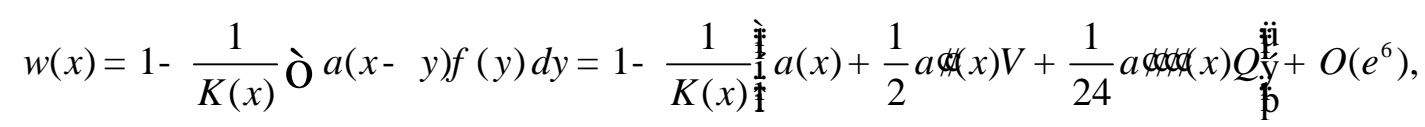

where $V=E_{f}\left[x^{2}\right]$ and $Q=E_{f}\left[x^{4}\right]$. We then expand $w(x)$ around $x=0$, assuming that the competition kernel and the carrying-capacity function are both symmetric around 0 , $a(x)=a(-x)$ and $K(x)=K(-x)$, and peaked at $1, a(0)=1$ and $K(0)=1$,

$$
\begin{aligned}
& w(x)=\frac{1}{2}\left\{K \Phi(0) x^{2}-a \Phi(0)\left(x^{2}+V\right)\right\} \\
& -\frac{1}{24}\left\{a d(0)\left(Q+6 V x^{2}+x^{4}\right)-6 a+(0) K(0)\left(V x^{2}+x^{4}\right)\right. \\
& \left.+6 K \Phi(0)^{2} x^{4}-K \operatorname{ded}(0) x^{4}\right\}+O\left(e^{6}\right) \text {. }
\end{aligned}
$$

Taking on both sides the expectation $E_{f}[\mathrm{~K}]$ with respect to $f(x)$ yields

$$
\begin{aligned}
& \bar{w}=\frac{1}{2}\{K \phi(0) V-2 a \phi(0) V\} \\
& -\frac{1}{24}\left\{a \operatorname{den}\left(2 Q+6 V^{2}\right)-6 a+(0) K \phi(0)\left(V^{2}+Q\right)\right. \\
& +6 K \Phi(0)^{2} Q-K
\end{aligned}
$$

which gives the selection differential

$$
\begin{aligned}
& w(x)-\bar{w}=\frac{1}{2}\{K \phi(0)-a \phi(0)\}\left(x^{2}-V\right) \\
& -\frac{1}{24}\left\{a \operatorname{ard}(0)-6 a \phi(0) K \phi(0)+6 K \Phi(0)^{2}-K d(0)\right\}\left(x^{4}-Q\right) \\
& -\frac{1}{4}\{a \notin(0)-a \notin(0) K \Phi(0)\} V\left(x^{2}-V\right)+O\left(e^{6}\right) \text {. }
\end{aligned}
$$

Substituting this result into eq. (A1) then yields 


$$
\begin{aligned}
\frac{d V}{d t}= & \frac{1}{2}\{K \Phi(0)-a \Phi(0)\}\left\{Q-V^{2}\right\} \\
& -\frac{1}{24}\left\{a d(0)-6 a \notin(0) K \Phi(0)+6 K \Phi(0)^{2}-K\right. \\
& -\frac{1}{4}\{a d(0)\}\{H-Q V\}
\end{aligned}
$$

where $H=E_{f}\left[x^{6}\right]$ is the sixth moment of the character distribution.

Without loss of generality, we can scale the character $x$ so that $a(0)=-1$. If the curvature of carrying capacity at $x=0$ is only slightly larger than $a \notin(0)$, we can set $K \Phi(0)=a \notin(0)+d=-1+d$, where $d$ is a small positive constant measuring the net disruptiveness of selection. Substituting these second derivatives into eq. (A6) and neglecting higher-order terms in $d$ (noting that the equilibrium morph variance $V$ for which disruptive and stabilizing selection pressures balance is of order $O(d)$, so that $Q=O\left(d^{2}\right)$ and $\left.H=O\left(d^{3}\right)\right)$ then yields

$$
\begin{aligned}
\frac{d V}{d t}= & \frac{1}{2} d\left\{Q-V^{2}\right\}-\frac{1}{2}\left\{Q-V^{2}\right\} V \\
& -\frac{1}{24} g_{K}\{H-Q V\}+\frac{1}{24} g_{a}\left(H+5 Q V-6 V^{3}\right)+O\left(e^{6}\right),
\end{aligned}
$$

where $g_{a}=3-a$ a $/ a \phi(0)^{2}$ and $g_{K}=3-K$ of competition kernel and carrying-capacity function, respectively (a positive value of these measures indicates a platykurtic function and a negative value indicates a leptokurtic function). For the functions specified in eq. (54), we obtain $d=1-w^{-2}, g_{a}=0$, and $g_{K}=24 g(1-d)^{-2} \gg 24 g$, which, when substituted in eq. (A7), recovers eq. (55) in the main text. 


\section{Literature cited}

Abrams, P. A., H. Matsuda, and Y. Harada. 1993. Evolutionarily unstable fitness maxima and stable fitness minima of continuous traits. Evolutionary Ecology 7:465-487.

Barton, N. H., and M. Turelli. 1991. Natural and sexual selection on many loci. Genetics 127:229-255.

Bonsall, M. B., V. A. A. Jansen, and M. P. Hassell. 2004. Life history trade-offs assemble ecological guilds. Science 306:111-114.

Boots, M., and Y. Haraguchi. 1999. The evolution of costly resistance in host-parasite systems. American Naturalist 153:359-370.

Boots, M., P. J. Hudson, and A. Sasaki. 2004. Large shifts in pathogen virulence relate to host population structure. Science 303:842-844.

Bowers, R. G., A. White, M. Boots, S. A. H. Geritz, and É. Kisdi. 2003. Evolutionary branching/speciation: contrasting results from systems with explicit or emergent carrying capacities. Evolutionary Ecology Research 5:883-891.

Brown, J. S., and N. B. Pavlovic. 1992. Evolution in heterogeneous environments: effects of migration on habitat specialization. Evolutionary Ecology 6:360-382.

Brown, J. S., and T. L. Vincent. 1987. A theory for the evolutionary game. Theoretical Population Biology 31:140-166.

Bull, J. J. 1987. Evolution of phenotypic variance. Evolution 41:303-315.

Bulmer, M. G. 1972. The genetic variability of polygenic characters under optimizing selection, mutation and drift. Genetical Research 19:17-25.

Bulmer, M. 1974. Density-dependent selection and character displacement. American Naturalist 108:45-58.

Bulmer, M. G. 1992. The mathematical theory of quantitative genetics. Oxford University Press, Oxford, UK.

Cheptou, P. O., and A. Mathias. 2001. Can varying inbreeding depression select for intermediary selfing rate? American Naturalist 157:361-373.

Chow, S. S., C. O. Wilke, C. Ofria, R. E. Lenski, and C. Adami. 2004. Adaptive radiation from resource competition in digital organisms. Science 305:84-86.

Claessen, D., and U. Dieckmann. 2002. Ontogenetic niche shifts and evolutionary branching in size-structured populations. Evolutionary Ecology Research 4:189-217.

Cohen, D., and S. A. Levin. 1991. Dispersal in patchy environments: the effect of temporal and spatial structure. Theoretical Population Biology 39:63-99.

Day, T. 2000. Competition and the effect of spatial resource heterogeneity on evolutionary diversification. American Naturalist 155:790-803.

Day, T., and K. A. Young. 2004. Competitive and facilitative evolutionary diversification. BioScience 54:101-109. 
De Jong, T., and S. A. H. Geritz. 2001. The role of geitonogamy in the gradual evolution towards dioecy in cosexual plants. Selection 2:133-146.

Dercole, F. 2003. Remarks on branching-extinction evolutionary cycles. Journal of Mathematical Biology 47:569-580.

Dieckmann, U., and M. Doebeli. 1999. On the origin of species by sympatric speciation. Nature 400:354-357.

Dieckmann, U., and M. Doebeli. 2005. Pluralism in evolutionary theory. Journal of Evolutionary Biology 18:1209-1213.

Dieckmann, U., and R. Law. 1996. The dynamical theory of coevolution: a derivation from stochastic ecological processes. Journal of Mathematical Biology 34:579-612.

Dieckmann. U., M. Doebeli, J. A. J. Metz, and D. Tautz eds. 2004. Adaptive Speciation. Cambridge University Press, Cambridge, UK.

Doebeli, M. 1996a. An explicit genetic model for ecological character displacement. Ecology 77:510-520.

Doebeli, M. 1996b. A quantitative genetic model for sympatric speciation. Journal of Evolutionary Biology 9:893-909.

Doebeli, M. 2002. A model for the evolutionary dynamics of cross-feeding polymorphisms in microorganisms. Population Ecology 44:59-70.

Doebeli, M., and U. Dieckmann. 2000. Evolutionary branching and sympatric speciation caused by different types of ecological interactions. American Naturalist 156(suppl.):S77-S101.

Doebeli, M., and U. Dieckmann. 2003. Speciation along environmental gradients. Nature 421:259-264.

Doebeli, M., C. Hauert, and T. Killingback. 2004. The evolutionary origin of cooperators and defectors. Science 306:859-862.

Doebeli, M., and G. D. Ruxton. 1997. Evolution of dispersal rates in metapopulation models: branching and cyclic dynamics in phenotype space. Evolution 51:1730-1741.

Doebeli, M., H. J. Blok, O. Leimar, and U. Dieckmann. 2007. Multimodal pattern formation in phenotype distributions of sexual populations. Proceedings of the Royal Society London B: Biological Sciences 274:347-357.

Egas, M., U. Dieckmann, and M. W. Sabelis. 2004. Evolution restricts the coexistence of specialists and generalists: the role of trade-off structure. American Naturalist 163:518-531.

Egas M., M. W. Sabelis, and U. Dieckmann. 2005. Evolution of specialization and ecological character displacement of herbivores along a gradient of plant quality. Evolution 59:507-520.

Ellner, S., and N. G. Hairston. 1994. Role of overlapping generations in maintaining geneticvariation in a fluctuating environment. American Naturalist 143:403-417. 
Ellner, S., and A. Sasaki. 1996. Patterns of genetic polymorphism maintained by fluctuating selection with overlapping generations. Theoretical Population Biology 50:31-65.

Ernande, B., and U. Dieckmann. 2004. The evolution of phenotypic plasticity in spatially structured environments: implications of intraspecific competition, plasticity costs and environmental characteristics. Journal of Evolutionary Biology 17:613-628.

Eshel, I. 1983. Evolutionary and continuous stability. Journal of Theoretical Biology 103:99-111.

Eshel, I., and U. Motro. 1981. Kin selection and strong evolutionary stability of mutual help. Theoretical Population Biology 19:420-433.

Falconer, D. S. 1996. Introduction to Quantitative Genetics. Longman Group, Harlow, UK.

Felsenstein, J. 1976. The theoretical population genetics of variable selection and migration. Annual Reviews of Ecology and Systematics 10:253-280.

Ferdy, J. B., L. Després, and B. Godelle. 2002. Evolution of mutualism between globeflowers and their pollinating flies. Journal of Theoretical Biology 217:219-234.

Ferrière, R., J. L. Bronstein, S. Rinaldi, R. Law, and M. Gauduchon. 2002. Cheating and the evolutionary stability of mutualisms. Proceedings of the Royal Society London B: Biological Sciences 269:773-780.

Fort, H., M. Scheffer, E. H. Van Nes. 2009. The paradox of the clumps mathematically explained. Theoretical Ecology 2:171-176.

Geritz, S. A. H., É. Kisdi, G. Meszéna, and J. A. J. Metz. 1998. Evolutionarily singular strategies and the adaptive growth and branching of the evolutionary tree. Evolutionary Ecology 12:35-57.

Geritz, S. A. H., E. Van der Meijden, and J. A. J. Metz. 1999. Evolutionary dynamics of seed size and seedling competitive ability. Theoretical Population Biology 55:324-343.

Gudelj, I., F. van den Bosch, and C. A. Gilligan. 2004. Transmission rates and adaptive evolution of pathogens in sympatric heterogeneous plant populations. Proceedings of the Royal Society London B: Biological Sciences 271:2187-2194.

Gyllenberg, M., and G. Meszéna. 2005. On the impossibility of coexistence of infinitely many strategies. Journal of Mathematical Biology 50:133-160.

Haraguchi, Y., and A. Sasaki. 1996. Host-parasite arms race in mutation modifications: indefinite escalation despite a heavy load? Journal of Theoretical Biology 183:121-137.

Haraguchi, Y., and A. Sasaki. 1997. Evolutionary pattern of intra-host pathogen antigenic drift: Effect of cross-reactivity in immune response. Philosophical Transactions of the Royal Society London B: Biological Sciences 352:11-20.

Iwanaga, A., and A. Sasaki. 2004. Evolution of hierarchical cytoplasmic inheritance in the plasmodial slime mold Physarum polycephalum. Evolution 58:710-722.

Iwasa, Y., A. Pomiankowski, and S. Nee. 1991. The evolution of costly mate preferences. II. The ‘handicap’ principle. Evolution 45:1431-1442. 
Jansen, V. A. A., and G. S. E. E. Mulder. 1999. Evolving biodiversity. Ecology Letters 2:379-386.

Johst, K., M. Doebeli, and R. Brandl. 1999. Evolution of complex dynamics in spatially structured populations Proceedings of the Royal Society London B: Biological Sciences 266:1147-1154.

Kamo, M., A. Sasaki, and M. Boots. 2007. The role of trade-off shapes in the evolution of parasites in spatial host populations: an approximate analytical approach. Journal of Theoretical Biology 244:588-596.

Kimura M., and J. F. Crow. 1964. The number of alleles that can be maintained in a finite population. Genetics 49:725-738.

Kisdi, É. 1999. Evolutionary branching under asymmetric competition. Journal of Theoretical Biology 197:149-162.

Kisdi, É. 2001. Long-term adaptive diversity in Levene-type models. Evolutionary Ecology Research 3:721-727.

Kisdi, É., and S. A. H. Geritz. 2001. Evolutionary disarmament in interspecific competition. Proceedings of the Royal Society London B: Biological Sciences 268: 2589-2594.

Kisdi, É., F. J. A. Jacobs, and S. A. H. Geritz. 2001. Red Queen evolution by cycles of evolutionary branching and extinction. Selection 2:161-176.

Koella, J. C., and M. Doebeli. 1999. Population dynamics and the evolution of virulence in epidemiological models with discrete host generations. Journal of Theoretical Biology 198:461-475.

Kondrashov, A. S., and L. Y. Yampolsky. 1996. High genetic variability under the balance between symmetric mutation and fluctuating stabilizing selection. Genetical Research 68:157-164.

Lande, R. 1975. The maintenance of genetic variability by mutation in a polygenic character with linked loci. Genetical Research 26:221-235.

Lande, R. 1976. Natural selection and random genetic drift in phenotypic evolution. Evolution 30:314-334.

Lande, R. 1979. Quantitative genetic-analysis of multivariate evolution, applied to brain body size allometry. Evolution 33:402-416.

Lande, R. 1981. Models of speciation by sexual selection on polygenic traits. Proceedings of the National Academy of Sciences of the United States of America 78:3721-3725.

Lande, R. 1982. A quantitative genetic theory of life history evolution. Ecology 63:607-615.

Lande, R. 1985. Expected time for random genetic drift of a population between stable phenotypic states. Proceedings of the National Academy of Sciences of the United States of America 82:7641-7645.

Lande, R. 1986. The dynamics of peak shifts and the pattern of morphological evolution. Paleobiology 12:343-354. 
Lande, R., and M. Kirkpatrick. 1988. Ecological speciation by sexual selection. Journal of Theoretical Biology 133:85-98.

Law, R., J. L. Bronstein, and R. Ferrière. 2001. On mutualists and exploiters: plant-insect coevolution in pollinating seed-parasite systems. Journal of Theoretical Biology 212:373-389.

Law, R., P. Marrow, and U. Dieckmann. 1997. On evolution under asymmetric competition. Evolutionary Ecology 11:485-501.

Leimar, O. 2005. The evolution of phenotypic polymorphism: randomized strategies versus evolutionary branching. American Naturalist 165:669-681.

Leimar, O., M. Doebeli, and U. Dieckmann. 2008. Evolution of phenotypic clusters through competition and local adaptation along an environmental gradient. Evolution 62:807-822.

Levin, S. A., D. Cohen, and A. Hastings. 1984. Dispersal strategies in patchy environment. Theoretical Population Biology 19:169-200.

Loeuille, N., and M. Loreau. 2005. Evolutionary emergence of size structured food webs. Proceedings of the National Academy of Sciences of the USA 102:5761-5766.

Ludwig, D., and S. A. Levin. 1991. Evolutionary stability of plant communities and the maintenance of multiple dispersal types. Theoretical Population Biology 40:285-307.

MacArthur, R. 1969. Species packing, and what interspecies competition minimizes. Proceedings of the National Academy of Sciences of the USA 64:1369-1371.

MacArthur, R. 1970. Species packing and competitive equilibrium for many species. Theoretical Population Biology 1:1-11.

MacArthur, R., and R. Levins. 1967. The limiting similarity, convergence, and divergence of coexisting species. American Naturalist 101:377-385.

Maire, N., M. Ackermann, and M. Doebeli. 2001. Evolutionary branching and the evolution of anisogamy. Selection 2:119-132.

Matessi, C., and S. D. Jayakar. 1981. Coevolution of species in competition - a theoretical study. Proceedings of the National Academy of Sciences of the USA 78:1081-1084.

Matsuda, H., and P. A. Abrams. 1999. Why are equally sized gametes so rare? The instability of isogamy and the cost of anisogamy. Evolutionary Ecology Research 1:769-784.

Mathias, A., and É. Kisdi. 2002. Adaptive diversification of germination strategies. Proceedings of the Royal Society London B: Biological Sciences 269:151-156.

Mathias, A., É. Kisdi, and I. Olivieri. 2001. Divergent evolution of dispersal in a heterogeneous landscape. Evolution 55:246-259.

May, R. M. 1973. Stability and Complexity in Model Ecosystems. Princeton University Press, Princeton, NJ.

May, R. M. 1974. On the theory of niche overlap. Theoretical Population Biology 5:297-332.

Maynard Smith, J. 1982. Evolution and the Theory of Games. Cambridge University Press, 
Cambridge, UK.

Meszéna, G., I. Czibula, and S. A. H. Geritz. 1997. Adaptive dynamics in a 2-patch environment: a toy model for allopatric and parapatric speciation. Journal of Biological Systems 5:265-284.

Meszéna, G., and E. Szathmáry. 2001. Adaptive dynamics of parabolic replicators. Selection 2:147-160.

Metz, J. A. J., S. A. H. Geritz, G. Meszéna, F. J. A. Jacobs, and J. S. van Heerwaarden. 1996. Adaptive dynamics: a geometrical study of the consequences of nearly faithful reproduction. Pages 183-231 in S. J. van Strien and S. M. Verduyn Lunel, eds. Stochastic and spatial structures of dynamical systems. North-Holland, Amsterdam, the Netherlands.

Metz, J. A. J., R. M. Nisbet, and S. A. H. Geritz. 1992. How should we define "fitness" for general ecological scenarios? Trends in Ecology \& Evolution 7:198-202.

Newman, C. M., J. E. Cohen, and C. Kipnis. 1985. Neo-darwinian evolution implies punctuated equilibria. Nature 315:400-401.

Parvinen, K. 1999. Evolution of migration in a metapopulation. Bulletin of Mathematical Biology 61:531-550.

Parvinen, K., and M. Egas. 2004. Dispersal and the evolution of specialisation in a two-habitat type metapopulation. Theoretical Population Biology 66:233-248.

Pigolotti, S., C. López, and E. Hernández-García. 2007. Species clustering in competitive Lotka-Volterra models. Physical Review Letters 98:258101.

Pigolotti, S., C. López, E. Hernández-García, and K. H. Andersen. 2009. How Gaussian competition leads to lumpy or uniform species distributions. Theoretical Ecology 3:89-96.

Rees, M., and M. Westoby. 1997. Game-theoretical evolution of seed mass in multi-species ecological models. Oikos 78:116-126.

Regoes, R. R., M. A. Nowak, and S. Bonhoeffer. 2000. Evolution of virulence in a heterogeneous host population. Evolution 54:64-71.

Reuter, M., K. R. Helms, L. Lehmann, and L. Keller. 2004. Effects of brood manipulation costs on optimal sex allocation in social Hymenoptera. American Naturalist 164:E73-E82.

Roughgarden, J. 1972. Evolution of niche width. American Naturalist 106:683-718.

Roughgarden, J. 1976. Resource partitioning among competing species - a coevolutionary approach. Theoretical Population Biology 9:388-424.

Rosenzweig, M. L. 1978. Competitive speciation. Biological Journal of the Linnean Society 10:275-289.

Sasaki, A. 1997. Clumped distribution by neighborhood competition. Journal of Theoretical Biology 186:415-430. 
Sasaki, A., and G. de Jong. 1999. Density dependence and unpredictable selection in a heterogeneous environment: compromise and polymorphism in the ESS reaction norm. Evolution 53:1329-1342.

Sasaki, A., and S. Ellner. 1995. The evolutionarily stable phenotype distribution in a random environment. Evolution 49:337-350.

Sasaki, A., and S. Ellner. 1997. Quantitative genetic variance maintained by fluctuating selection with overlapping generations: variance components and covariances. Evolution 51:682-696.

Sasaki, A., and H. C. J. Godfray. 1999. A model for the coevolution of resistance and virulence in coupled host-parasitoid interactions. Proceedings of the Royal Society London B 266:455-463.

Schreiber, S. J., and G. A. Tobiason. 2003. The evolution of resource use. Journal of Mathematical Biology 47:56-78.

Slatkin, M. 1979. Frequency-dependent and density-dependent selection on a quantitative character. Genetics 93:755-771.

Slatkin, M. 1980. Ecological character displacement. Ecology 61:163-177.

Slatkin, M., and R. Lande. 1976. Niche width in a fluctuating environment-density independent model. American Naturalist 110:31-55.

Szabó, P., and G. Meszéna. 2006. Limiting similarity revisited. Oikos 112:612-619.

Taper, M. L., and T. J. Case. 1985. Quantitative genetic models for the coevolution of character displacement. Ecology 66:355-371.

Taylor, P., and T. Day. 1997. Evolutionary stability under the replicator and the gradient dynamics. Evolutionary Ecology 11:579-590.

Troost, T. A., B. W. Kooi, and S. A. L. M. Kooijman. 2005. Ecological specialization of mixotrophic plankton in a mixed water column. American Naturalist 166:E45-E61.

Turelli, M. 1984. Heritable genetic variation via mutation-selection balance: Lerch's zeta meets the abdominal bristle. Theoretical Population Biology 25:138-193.

Van der Laan, J. D., and P. Hogeweg. 1995. Predator-prey coevolution: interactions across different timescales. Proceedings of the Royal Society London B: Biological Sciences 259:35-42.

Van Dooren, T. J. M., and O. Leimar. 2003. The evolution of environmental and genetic sex determination in fluctuating environments. Evolution 57:2667-2677.

Van Doorn, G. S., P. C. Luttikhuizen, and F. J. Weissing. 2001. Sexual selection at the protein level drives the extraordinary divergence of sex-related genes during sympatric speciation. Proceedings of the Royal Society London B: Biological Sciences 268:2155-2161.

Van Doorn, G. S., U. Dieckmann, and F. J. Weissing. 2004. Sympatric speciation by sexual selection: a critical re-evaluation. American Naturalist 163:709-725. 
Vincent, T. L., Y. Cohen, and J. S. Brown. 1993. Evolution via strategy dynamics. Theoretical Population Biology 44:149-176.

Whitlock, M. C. 1995. Variance-induced peak shifts. Evolution 49:252-259.

Whitlock, M. C. 1997. Founder effects and peak shifts without genetic drift: adaptive peak shifts occur easily when environments fluctuate slightly. Evolution 51:1044-1048. 


\section{Figures}

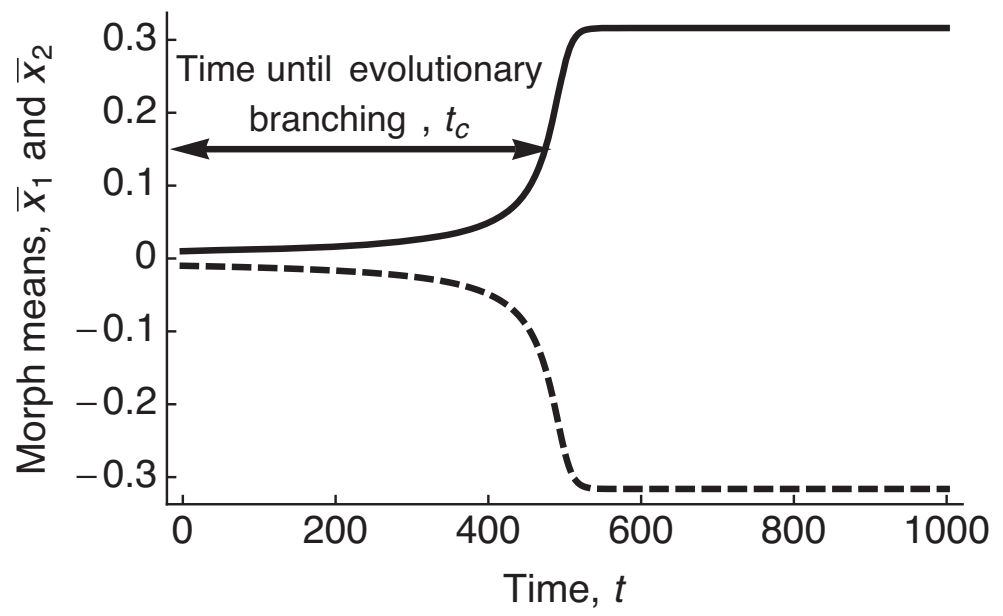

Figure 1. Evolutionary branching as described by the oligomorphic dynamics of two morphs (continuous curves: morph 1; dashed curves: morph 2) for a Gaussian competition kernel $a(x)=\exp \left(-\frac{1}{2} x^{2} / s^{2}\right)$ with $s=1$ and a Gaussian resource distribution $K(x)=\exp \left(-\frac{1}{2} x^{2} / w^{2}\right)$ with $w=1.054$. The net disruptiveness at $x=0$ thus equals $d=K(0)-a(0)=0.1>0$, so a monomorphism at $x=0$ is not evolutionarily stable. Variance dynamics are based on the Gaussian closure, $Q_{i}=3 V_{i}^{2}$. The dynamics of the means and variances of morph $i=1,2$ are given by $d \bar{x}_{i} / d t=V_{i} \bar{x}_{i}\left(d-\bar{x}_{i}^{2}\right)$ and $d V_{i} / d t=V_{i}^{2}\left(d-\bar{x}_{i}^{2}\right)$, which are obtained from eq. (52)-(53) for $\gamma_{a}=\gamma_{K}=0$. These start from a symmetric dimorphism with $p_{1}(0)=p_{2}(0)=0.5, \bar{x}_{1}(0)=-\bar{x}_{2}(0)=0.01$, and $V_{1}(0)=V_{2}(0)=0.02$. The time to evolutionary branching is approximated by $t_{c}=1 /(d V(0))=500$ (double-headed arrow), in good agreement with the actually observed duration of evolutionary branching. 
a

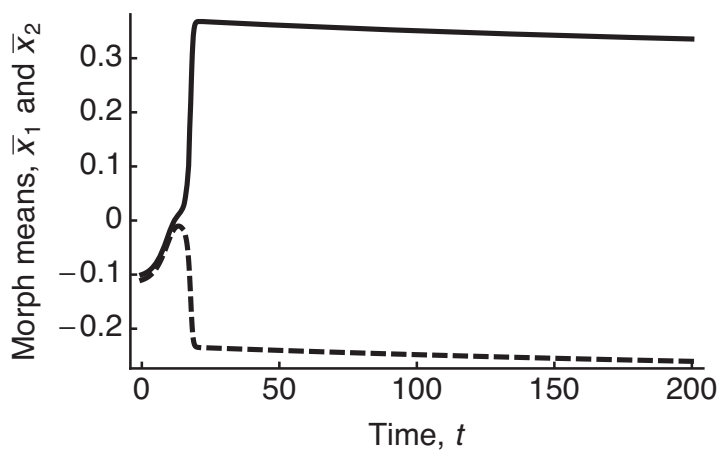

b

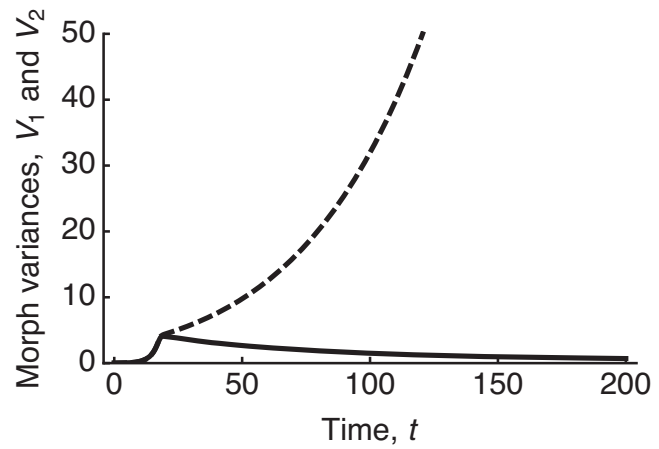

C

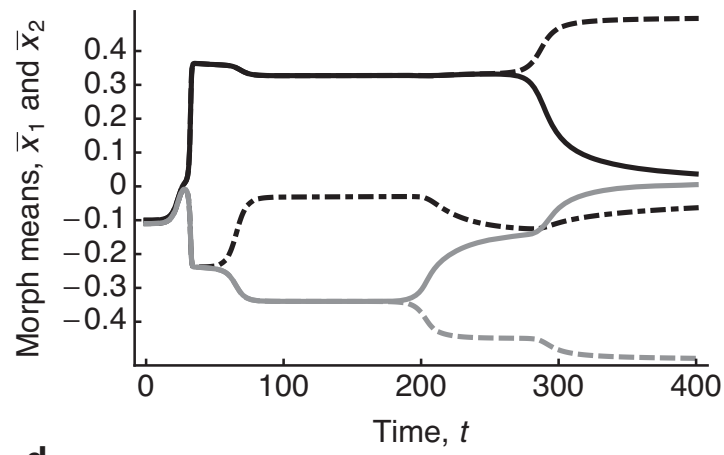

d

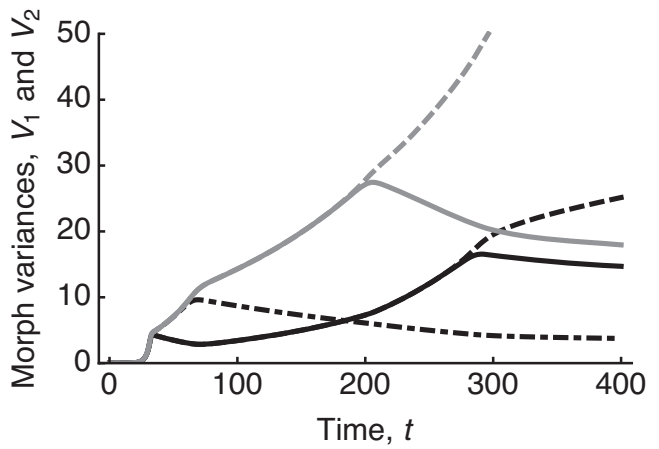

Figure 2. Oligomorphic dynamics for a Gaussian competition kernel $a(x)=\exp \left(-\frac{1}{2} x^{2} / s^{2}\right) \quad$ with $s=1$ and a Gaussian resource distribution $K(x)=\exp \left(-\frac{1}{2} x^{2} / w^{2}\right)$ with $w=1.1$. Variance dynamics are based on the house-of-card closure with $c_{4} / c_{2}=2$, resulting in $Q_{i}=2 V_{i}$. Since $w>s$, a monomorphism at $x=0$ is not evolutionarily stable. (a, b) Dynamics of morph means (a) and morph variances (b) for two morphs ( $n=2$; continuous curves: morph 1; dashed curves: morph 2) for initial conditions $p_{1}(0)=0.4, p_{2}(0)=0.6, \bar{x}_{1}(0)=-0.1, \bar{x}_{2}(0)=-0.11$, and $V_{1}(0)=V_{2}(0)=0.01$. (c, d) Dynamics of morph means (c) and morph variances (d) for five morphs ( $n=5$; continuous black curves: morph 1; dashed black curves: morph 2; dot-dashed black curves: morph 3; continuous gray curves: morph 4; dashed gray curves: morph 5) for initial conditions $p_{i}(0)=1 / 5, \quad \bar{x}_{1}(0)=-0.1, \quad \bar{x}_{2}(0)=\bar{x}_{1}(0)+10^{-8}, \quad \bar{x}_{3}(0)=-0.11, \quad \bar{x}_{4}(0)=\bar{x}_{3}(0)-10^{-5}$, $\bar{x}_{5}(0)=\bar{x}_{4}(0)-10^{-13}$, and $V_{i}(0)=10^{-4}$ for $i=1, \mathrm{~K}, 5$. 

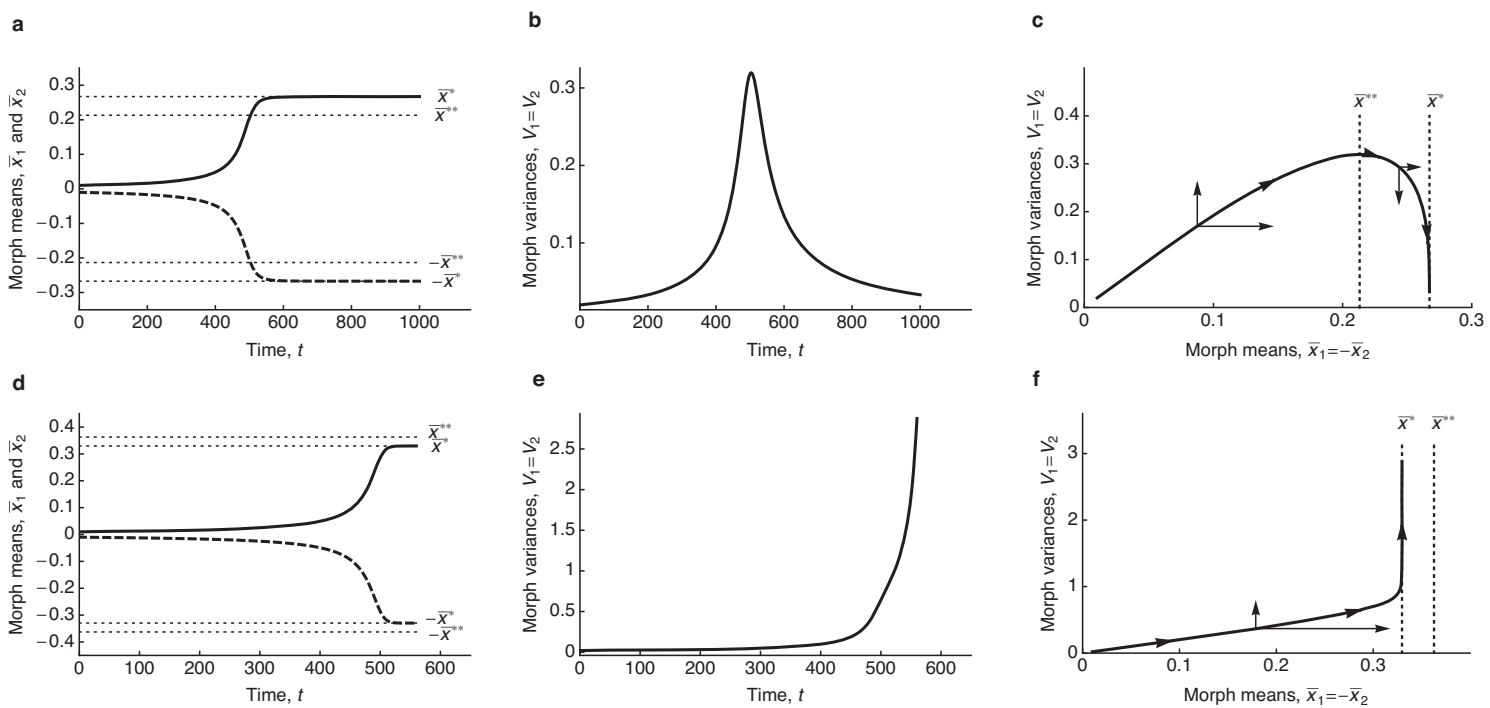

Figure 3. Oligomorphic dynamics of a symmetric dimorphism with $p_{1}(t)=p_{2}(t)=0.5$, $\bar{x}_{1}(t)=-\bar{x}_{2}(t)$, and $V_{1}(t)=V_{2}(t)$ for a Gaussian competition kernel $a(x)=\exp \left(-\frac{1}{2} x^{2} / s^{2}\right)$ with $s=1$ and a potentially platykurtic resource distribution $K(x)=\exp \left(-\frac{1}{2} x^{2} / w^{2}-g x^{4}\right)$ with $g=\frac{1}{24} g_{K}$ (continuous curves: morph 1; dashed curves: morph 2). The net disruptiveness is set to $d=K \Phi(0)-a \notin(0)=s^{-2}-w^{-2}=0.1>0$, which implies $w » 1.054$, so a monomorphism at $x=0$ is not evolutionarily stable. Variance dynamics are based on the Gaussian closure, $Q_{i}=3 V_{i}^{2}$. (a, b, c) When the resource distribution is more platykurtic than the competition kernel, $g_{K}=2.4>g_{a}=0$, the isocline $x=\bar{x}^{* *}=\sqrt{d /\left(1+\frac{1}{2} g_{K}-g_{a}\right)}$ $=0.213$, along which $d V_{1} / d t=d V_{2} / d t=0$, is situated to the left side of the isocline $x=\bar{x}^{*}=\sqrt{d /\left(1+\frac{1}{6} g_{K}-\frac{2}{3} g_{a}\right)}=0.267$, along which $d \bar{x}_{1} / d t=d \bar{x}_{2} / d t=0 \quad$ (c). This means that the dynamics converge to a stable dimorphism with morph means $\bar{x}_{1}=\bar{x}^{*}, \bar{x}_{2}=-\bar{x}^{*}$ (a), and vanishing morph variances $V_{1}=V_{2}=0$ (b). (d, e, f) When the competition kernel is more platykurtic than the resource distribution, $g_{K}=-0.48<g_{a}=0$, the trajectory instead reaches the isocline $x=\bar{x}^{*}=0.330$ before it has the possibility to reach the isocline $x=\bar{x}^{* *}=0.363$ (f). This means that the morph variances keep growing (e) even after the morph means have already become stationary, at $x=\bar{x}^{*}$ (d), resulting in an unlimited explosion of the two morph variances. 
a
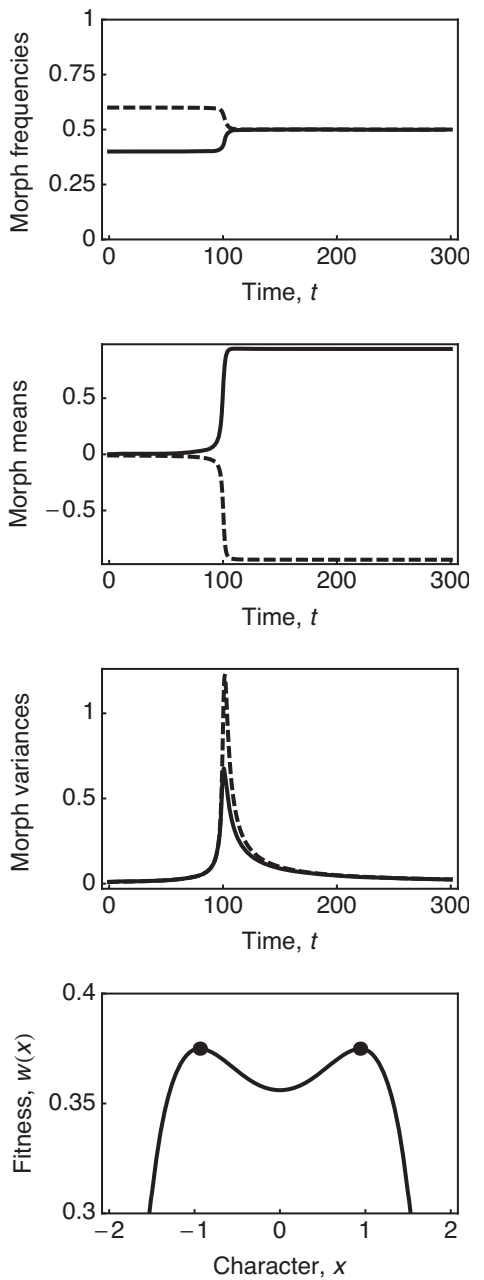

b
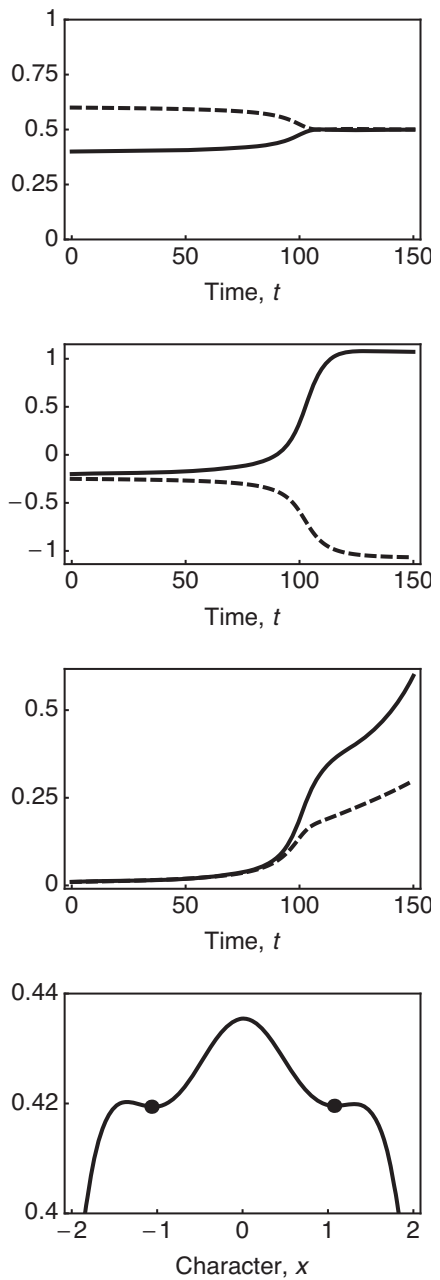
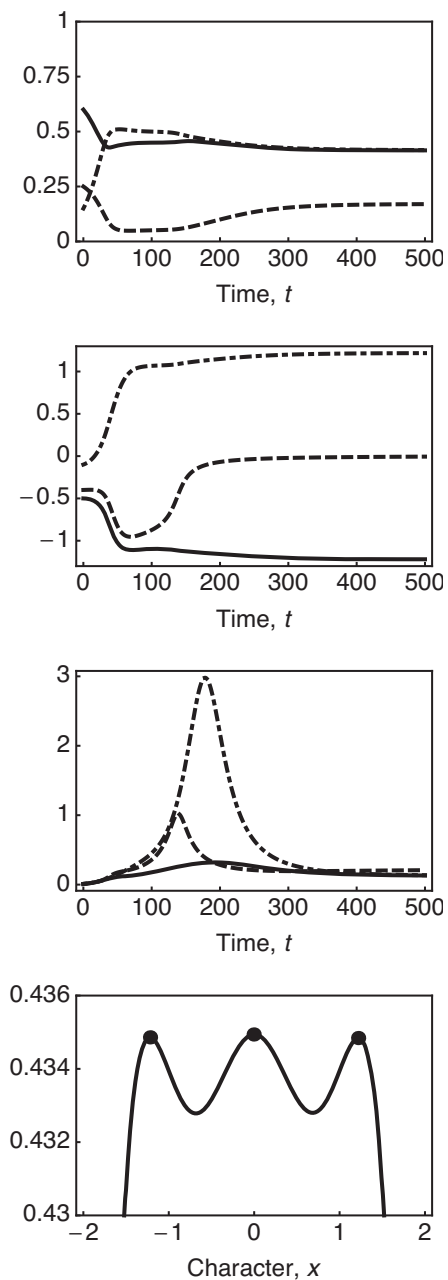

Figure 4. Oligomorphic dynamics for a Gaussian competition kernel $a(x)=\exp \left(-\frac{1}{2} x^{2} / s^{2}\right)$ with $s=1$ and a quartic (and thus platykurtic) resource distribution $K(x)=\exp \left(-\frac{1}{12} x^{4} / h^{4}\right)$ (top row: morph frequencies; second row: morph means; third row: morph variances; fourth row: fitness landscape $w(x)=1-\stackrel{\circ}{\mathrm{a}}_{i} p_{i} a\left(x-\bar{x}_{i}\right) / K(x)$ at the end of the shown time series). Variance dynamics are based on the Gaussian closure, $Q_{i}=3 V_{i}^{2}$. Oligomorphic analysis reveals that a monomorphism at $x=0$ is never evolutionarily stable, and that a symmetric dimorphism around $x=0$ is evolutionarily stable if $h / s<1.16$ (Figure 5). (a) Two-morph dynamics for $h / s=1$ and $m^{2}=0 \quad(n=2$; continuous curves: morph 1; dashed curves: morph 2). Starting from initial conditions $p_{1}(0)=0.4$, $p_{2}(0)=0.6, \bar{x}_{1}(0)=0.001, \bar{x}_{2}(0)=-0.01$, and $V_{1}(0)=V_{2}(0)=0.01$, a convergence stable and evolutionarily stable protected dimorphism emerges. (b) Two-morph dynamics for $h / s=1.2$ and $\mathrm{mm}^{2}=0 \quad(n=2$; continuous curves: morph 1; dashed curves: morph 2$)$. Starting from initial conditions $p_{1}(0)=0.4, p_{2}(0)=0.6, \bar{x}_{1}(0)=-0.2, \bar{x}_{2}(0)=-0.25$, and $V_{1}(0)=V_{2}(0)=0.01$, morph frequencies and morph means approach an evolutionarily singular symmetric dimorphism, but morph variances expand to infinity. (c) Three-morph 
dynamics for $h / s=1.2$ and $\mathrm{mm}^{2}=0.001(n=3$; continuous curves: morph 1 ; dashed curves: morph 2; dot-dashed curves: morph 3). Starting from initial conditions $p_{1}(0)=0.6$, $p_{2}(0)=0.25, \quad p_{3}(0)=0.15, \quad \bar{x}_{1}(0)=-0.5, \quad \bar{x}_{2}(0)=-0.4, \quad \bar{x}_{3}(0)=-0.1, \quad$ and $V_{1}(0)=V_{2}(0)=V_{3}(0)=0.01$, the population is evolutionarily stabilized by a secondary evolutionary branching between morphs 2 and 3: eventually all morph variances become stationary, since all morph means are situated at local maxima of the fitness landscape. 
a

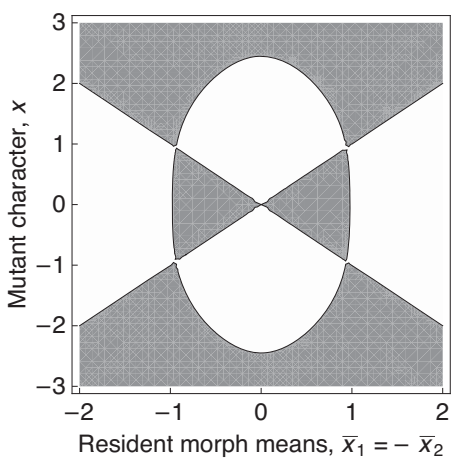

b

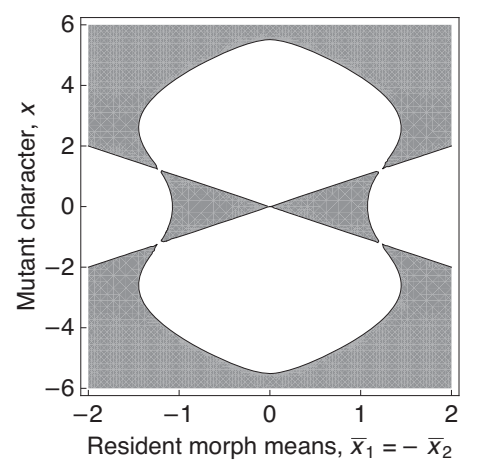

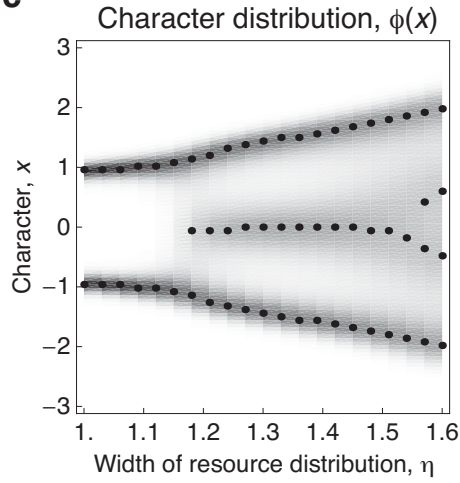

Figure 5. Evolutionary invasion analysis of a symmetric dimorphism with $p_{1}(t)=p_{2}(t)=0.5, \bar{x}_{1}(t)=-\bar{x}_{2}(t)$, and $V_{1}(t)=V_{2}(t)=0$ for a Gaussian competition kernel $a(x)=\exp \left(-\frac{1}{2} x^{2} / s^{2}\right)$ with $s=1$ and a quartic (and thus platykurtic) resource distribution $K(x)=\exp \left(-\frac{1}{12} x^{4} / h^{4}\right)$. (a) For $h=1<1.16$, the symmetric dimorphism with $\bar{x}_{1}(t)=-\bar{x}_{2}(t)=1$ is convergence stable and (globally) evolutionarily stable (white regions: mutant can invade; gray regions: mutant cannot invade). (b) For $h=1.5>1.16$, the symmetric dimorphism is convergence stable for $\bar{x}_{1}(t)=-\bar{X}_{2}(t) » 1.2$, but is not (neither locally, nor globally) evolutionarily stable (white regions: mutant can invade; black regions: mutant cannot invade). (c) Character distributions $f(x)$ resulting from oligomorphic dynamics based on 101 equally spaced character values in the range $-3<x<3$ for different widths $h$ of the resource distribution. The initial character distribution is Gaussian with a mean of 0.1 and a variance of 0.1 . The mutation rate between adjacent character values, which differ by $\mathrm{D} x=0.06$, is $m=2.8 \times 10^{-4}$, giving rise to the mutation variance $\mathrm{mm}^{2}=m E\left[(\mathrm{D} x)^{2}\right]=1 \times 10^{-6}$. The panel shows $f^{0.5}(x)$ (so as to improve visibility of low densities; white: 0; black: maximum value; with linear grayscales in between) together with morph means (filled circles) at $t=2,000$ for 21 values of $h$. 
a
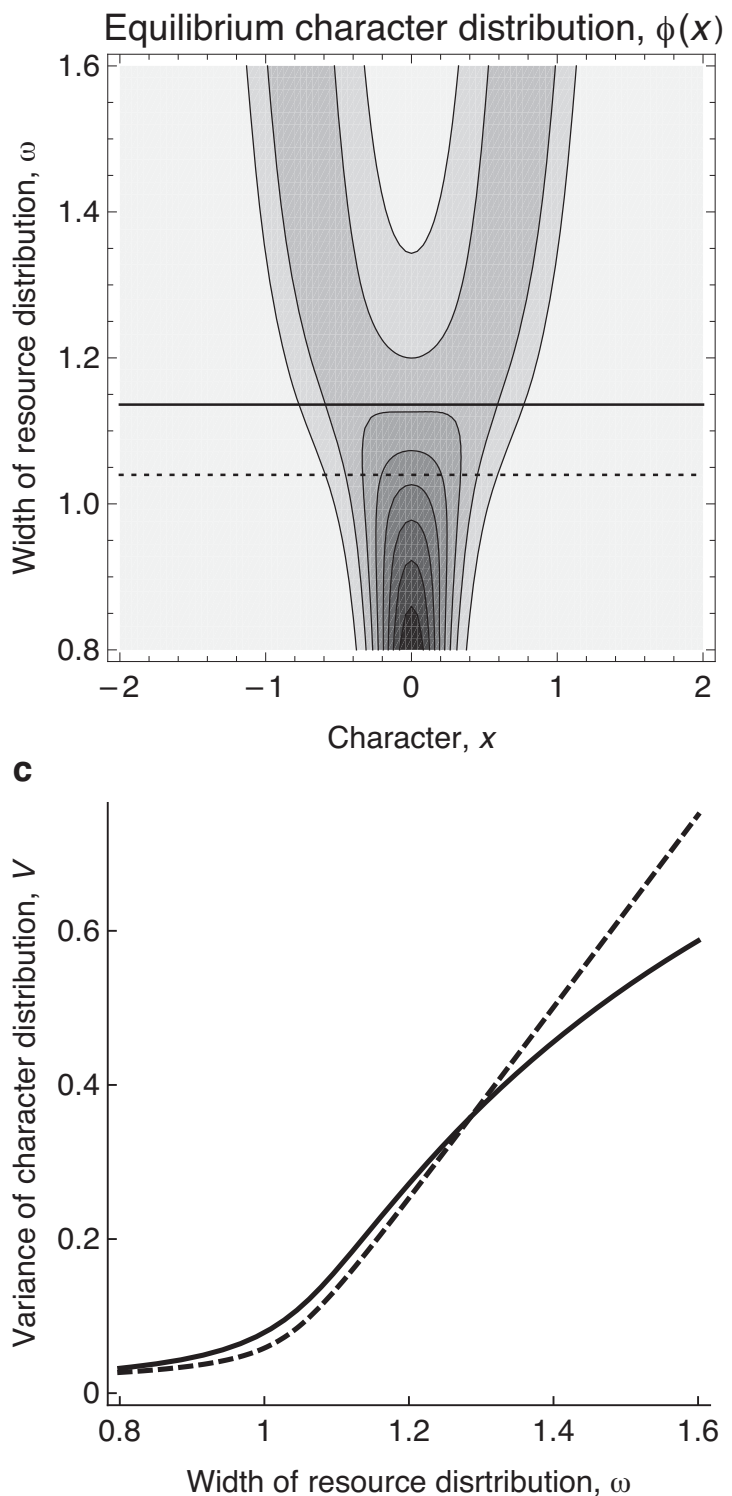

b

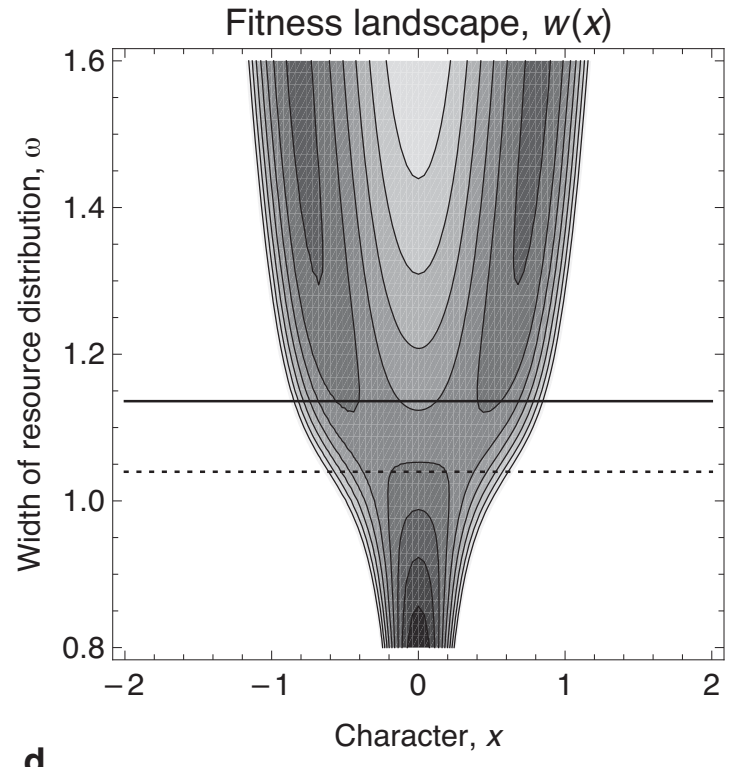

d

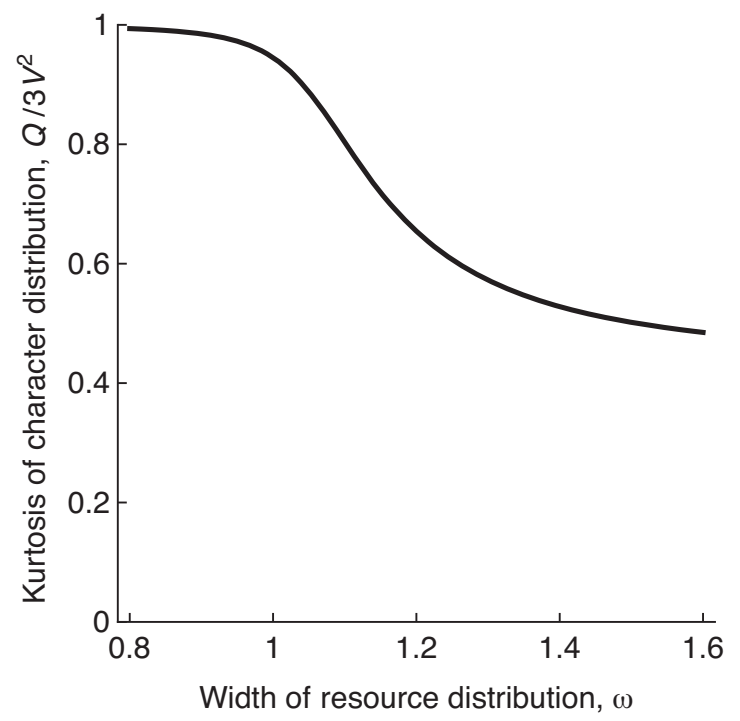

Figure 6. Effects of mutation on evolutionary branching for a Gaussian competition kernel $a(x)=\exp \left(-\frac{1}{2} x^{2} / s^{2}\right)$ with $s=1$ and a potentially platykurtic resource distribution $K(x)=\exp \left(-\frac{1}{2} x^{2} / w^{2}-g x^{4}\right)$ with $g=0.05$. Without mutation, the threshold condition for evolutionary branching is $w / s=1$. (a) Equilibrium character distributions $f(x)$ resulting from oligomorphic dynamics based on 101 equally spaced character values in the range $-2<x<2$ for different widths $w$ of the resource distribution. The mutation rate between adjacent character values, which differ by $\mathrm{D} x=0.04$, is $m=0.2$, giving rise to the mutation variance $m m^{2}=m E\left[(\mathrm{D} x)^{2}\right]=3.2 \times 10^{-4}$. With such mutation, the population remains unimodal for $1<w / s<1.136$ (continuous horizontal lines). (b) Fitness landscapes $w(x)$ for different widths $w$ of the resource distribution. With mutation, fitness landscapes remain unimodal for $1<w / s<1.040$ (dotted horizontal line), but become bimodal earlier than the charac- 
ter distribution (continuous horizontal lines) as $w$ is increased. (c) Variances of the equilibrium character distribution for different widths $w$ of the resource distribution. The continuous line represents the numerical results from panel (A), while the dashed line represents the approximation from eq. (57). (d) Kurtoses $Q /\left(3 V^{2}\right)=E\left[(\mathrm{D} x)^{4}\right] /\left(3 V^{2}\right)$ of the equilibrium character distribution for different widths $w$ of the resource distribution. As $w$ is increased above $1, Q /\left(3 V^{2}\right)<1$, so the shape of the equilibrium character distribution changes from Gaussian to platykurtic. 